\title{
Role of Seasonality on Predator-Prey-Subsidy Population Dynamics
}

\author{
Dorian Levy, Heather A. Harrington, and Robert A. Van Gorder* \\ Mathematical Institute, University of Oxford, Andrew Wiles Building, Radcliffe Observatory Quarter, Woodstock Road, Oxford, OX2 6GG, UK \\ *Robert.VanGorder@maths.ox.ac.uk
}

\begin{abstract}
The role of seasonality on predator-prey interactions in the presence of a resource subsidy is examined using a system of nonautonomous ordinary differential equations (ODEs). The problem is motivated by the Arctic, inhabited by the ecological system of arctic foxes (predator), lemmings (prey), and seal carrion (subsidy). We construct two nonlinear, nonautonomous systems of ODEs named the Primary Model, and the $n$-Patch Model. The Primary Model considers spatial factors implicitly, and the $n$-Patch Model considers space explicitly as a "Stepping Stone" system. We establish the boundedness of the dynamics, as well as the necessity of sufficiently nutritional food for the survival of the predator. We investigate the importance of including the resource subsidy explicitly in the model, and the importance of accounting for predator mortality during migration. We find a variety of non-equilibrium dynamics for both systems, obtaining both limit cycles and chaotic oscillations. We were then able to discuss relevant implications for biologically interesting predator-prey systems including subsidy under seasonal effects. Notably, we can observe the extinction or persistence of a species when the corresponding autonomous system might predict the opposite.
\end{abstract}

Keywords: predator-prey dynamics; allochthonous resource subsidy; population dynamics; non-equilibrium dynamics; chaotic oscillations

\section{Introduction}

\subsection{Evolution Of Species Interaction Models}

The interaction between coexisting species is an important issue in ecology, often leading to a mathematical description. Understanding these systems can help in understanding and preventing extinction, for instance by advising patrol teams on deterring poachers of endangered animals in Africa (Plumptre et al., 2014); or preventing the spread of illness such as controlling dengue disease using genetically modified mosquitoes (de Valdez et al., 2011).

One of the earliest theories of population dynamics is the Malthus-Verhulst logistic theory, argued by Malthus in his Essay on the Principle of Population (Malthus and Appleman, 1976) in 1798 and developed into a mathematical theory by Verhulst in 1838 (Berryman, 1992; Verhulst, 1838). The theory assumes that the resources available to a species remain more or less constant, whilst the species reproduces at a logarithmic rate. Eventually, the population size is too large to be sustained by the resource supply, and this hinders population growth. This gives rise to the Logistic Equation. The Logistic Equation was later derived from first principles by Lotka in 1925 (Berryman, 1992; Lotka, 1925), and was used in a model to describe the competition between two species competing for the same resources (Ayala et al., 1973).

Lotka and Volterra later derived the first model of predatorprey interactions, known as the Lotka-Volterra Equations (Berryman, 1992; Abrams, 2000; Volterra, 1931). Their model was based on the chemical principle of mass action. This model unrealistically predicts that the prey will grow without bound in the absence of predator. Surprisingly, Lotka did not use the Logistic Equation to describe the growth of the prey. However, inclusion of the Logistic Equation prevents the prey from growing unboundedly and therefore is often used in variants of the Lotka-Volterra Equations.

One variation to the Lotka-Volterra Equations was suggested by Solomon (1949), and a decade later by Holling $(1959,1966)$. Motivated by the fact that there is a limit to the amount of prey which can be consumed by a predator in a finite amount of time, they proposed a form identical to the Michaelis-Menten Equation (Berryman, 1992; Nevai and Van Gorder, 2012; Abrams, 2000). That model was then extended in Nevai and Van Gorder (2012) to investigate a system in which there exists an alternative food source for the predator in the form of a resource subsidy. This subsidy is the carrion of the prey in an independent predator-prey interaction. Conditions under which equilibria for that model exist were derived, and it was shown that there exist positive equilibria and limit cycles under appropriate conditions.

Other models of species interactions include the onepredator, two-prey model (Hutson and Vickers, 1982). This model is interesting because Gause's law states that two identical species cannot coexist unless they exploit their environment differently (Vance, 1978). Introducing a predator to a system of two identical prey populations allows them to coexist (Vance, 1978). Necessary conditions under which the three populations can coexist permanently, also known as 'permanent persistence', are found in Hutson and Vickers (1982). For permanent persistence, the populations can approach a positive equilibrium, follow a limit cycle, or even obey chaotic dynam- 
ics (Hutson and Vickers, 1982). Demonstrations of chaotic dynamics for the one-predator, two-prey system are given in Klebanoff and Hastings (1994).

Discrete-space models that investigate the effect the animal populations' migration often fall into one of two categories: "Island" models (Kareiva et al., 1990; Holmes et al., 1994) and "Stepping Stone" models (Kareiva et al., 1990; Levin, 1976).

The "Island" models include a set of patches in which different populations live. There is an assumed accessibility between all patches, and migration between patches occurs instantaneously (Kareiva et al., 1990). As such, spatial dimensions are not explicitly included in the model. An advantage of this class of model is that it is the most analytically accessible of the three classes (Kareiva et al., 1990). This class is used in the two-patch predator-prey-subsidy model in Nevai and Van Gorder (2012), and in models which investigating the effect of having a refuge for the prey (Sih, 1984). "Island" models typically use ordinary differential equations (ODEs) and often consider linear inter-patch diffusion (Levin, 1976; Kareiva et al., 1990; Nevai and Van Gorder, 2012).

The "Stepping Stone" classification of model is much like the "Island" class, however the patches are assigned spatial coordinates and as such, spatial dimensions are now explicitly included in the model (Kareiva et al., 1990). These models are particularly good at contrasting long-range and short-range effects and are the best models to apply to field data (Kareiva et al., 1990). "Stepping Stone" models are used in Lima (2002) which investigates predator migration using game theory to examine how the predator and prey should behave when choosing their locations.

\subsection{The Arctic Ecosystem}

The motivation for this study is the Arctic ecosystem. The Arctic is home to a unique ecosystem comprising of terrestrial and marine animals and plant-life. One of the species which lives in the Arctic is the arctic fox (Alopex lagopus), which is a predator. The arctic fox has a diet of lemmings (Cricetidae family), and certain migratory birds and bird eggs (Roth, 2002, 2003). They also scavenge seal (Phocidae family) carrion, a nutritional subsidy discarded by polar bears (Ursus martimus) (Roth, 2002; Nevai and Van Gorder, 2012), and hunt ringed seal (Phoca hispida) pubs in their birth lairs (Roth, 2002, 2003). The lemmings and the seal carrion are the main food sources for the arctic fox (Roth, 2003).

According to Audet et al. (2002), arctic foxes are born in litters with size ranging from 3 to 25 pups, depending on the location, as well as on how much food is available during that season. The foxes leave their den after a month. By the age of 9-10 months, the fox reaches sexual maturity and is considered to be an adult. They are a monogamous species, and mate for life. The age expectancy for the arctic fox is 3-4 years in the wild (Audet et al., 2002; Roth, 2003).

The dynamics of the lemming population size oscillates (Roth, 2002, 2003; Turchin et al., 2000; Fuller et al., 1975; Lindström et al., 2001) with peaks about every 3-4 years (Fuller et al., 1975). The reasons for this remain largely a mystery.
Graphs of lemming population size tend to have sharp peaks (Turchin et al., 2000) which is a feature characteristic of predators rather than prey. These peaks suggest that lemmings actively predate their plant food sources. Another possibility for these oscillations is that they are caused by climactic variations (Lindström et al., 2001), suggested by Elton in 1924 who noted coinciding fluctuations in several northern animals (Lindström et al., 2001). Elton rejected the possibility of these fluctuations being caused by lunar or volcanic activity because lunar activity occurs either on a much smaller or much larger time scale than the fluctuations, and volcanic eruptions occur too infrequently (Lindström et al., 2001). Consequently, solar activity is the only remaining candidate able to account for lemming fluctuations. As a result, seasonality is likely to be an important factor for the Arctic ecosystem.

The average daily temperature in the arctic in 2014 ranged from $242 \mathrm{~K}\left(-31^{\circ} \mathrm{C}\right)$ in the winter to $274 \mathrm{~K}\left(1^{\circ} \mathrm{C}\right)$ in the summer (Danish Meteorological Institute, 2015). This vast seasonal change in temperature has a large effect on the ecosystem by changing the environment and consequently the diets of the species inhabiting it.

The temperature also changes on a longer timescale. The past century saw an increase in the average yearly global temperature of approximately $0.6 \mathrm{~K}$ (Root et al., 2003). Consequently, the area occupied by sea ice in 2008 had shrunk to half the size of half a century earlier - a loss of one million square miles of ice (Borgerson, 2008). This seriously endangers the life of the terrestrial animals, and consumers of terrestrial animals, since the habitat is rapidly disappearing.

A study on polar bears in the gulf of Boothia described the effect of the melting of the arctic in the summer, and the freezing in the winter (Thiemann et al., 2008). It was found that in the spring-summer seasons, when the ice has receded, species such as the beluga whale (Delphinapterus leucas) and the narwhal (Monodon monoceros) become available as a food source and so form a large part of the polar bears' diet. However, in the autumn-winter when the ice freezes, polar bears shift their diet towards the ringed seals (Roth, 2003; Thiemann et al., 2008). Polar bears prefer the blubber of seals and so leave a substantial amount of the seal carcass for scavenging animals (Roth, 2003). During years in which there is an abundance of seal carcasses, the polar bears can be more selective consumers and therefore discard more of the seal remains. Accordingly, there are more seal carrion available during the autumn-winter period, compared to the spring-summer period, which consequently influences arctic fox behaviour.

Temperature is a leading contributor to the rate of decay of an animal corpse (Meyer et al., 2013; Talent, 2012) with decomposition taking place most rapidly when the temperature is highest. Therefore, the seal carrion is preserved during the winter when its production rate is high, however quickly decays during the summer. Rising global temperatures would mean an increase in the average decomposition rate of the carrion and therefore less availability as a subsidy for the arctic fox.

The change in the abundance of plant life and the change in temperature affect the growth rate of the lemmings. As suggested in Turchin and Hanski (1997), the intrinsic growth rate 
and the carrying capacity of the lemmings oscillate sinusoidally in a coupled manor such that the growth rate is largest in summer and smallest in the winter, while competition between the lemmings remains constant throughout the year.

Therefore, the arctic fox is likely to have a diet rich in seal carrion during the winter when there is an abundance of the subsidy and the lemming population is low, and a diet rich in lemming during the summer when the opposite is true.

The arctic fox is a migratory animal, which travels from areas in which the lemmings breed to areas in which polar bears live. The arctic fox migrates every 3-4 years in September by following random paths, rather than developing repetition (Wrigley and Hatch, 1976). Migration usually finishes by midFebruary when the breeding season begins. During migration, there is a high death rate due to lack of food sources, trapping, and diseases such as rabies which can affect up to three-quarters of the population (Wrigley and Hatch, 1976). During winters when migration does not occur, starvation is still a key factor in the death rate (Prestrud, 1991).

\subsection{Overview}

We consider the predator-prey-subsidy (PPS) model motivated by the interaction between arctic foxes (predator), lemmings (prey), and seal carrion (subsidy). The lemming and the seal carrion are the primary components in the diet of the arctic fox (Roth, 2003) and therefore we focus on the effect of these two food sources on the ecosystem. In Section 2, we introduce the mathematical model that we use to investigate PPS dynamics. The equations are nonautonomous in order to account for seasonality. We develop a Primary Model, where we do not consider spatial dimensions. Then, we develop an $n$-Patch Model where the prey and subsidy are confined to disjoint regions, however the predator is permitted to travel between these regions. In Section 3, we investigate the effect of seasonality on the dynamics of the PPS populations in the Primary Model. These results are then extended in Section 4 where we investigate the effect of seasonality on the PPS populations in the $n$-Patch Model. In Section 5 we investigate chaotic behaviour presented in both models. Then we discuss the biological implications of the results attained in Section 6, and we give a summary of the results along with conclusions in Section 7.

\section{The Predator-Prey-Subsidy Model}

In this section, we derive two distinct models for predatorprey-subsidy interactions. Our models are motivated by interactions in the Arctic between arctic foxes (predator), lemmings (prey), and seal carrion (subsidy). With minor adaptions, the models we develop are applicable to other ecosystems with PPS interactions.

The first of our models, named the Primary Model, is a three dimensional system of nonlinear, nonautonomous, ODEs. Whilst it has no explicit spatial-dependence, spatial effects are included in the form of a higher mortality rate during the predators' migratory period. The Primary Model is an example of an "Island" model. The second model, named the $n$-Patch Model, explicitly considers space by considering $n$ patches of predator, prey, and subsidy between which predator inter-territorial migration occurs. This model has $3 n$ dimensions. The $n$-Patch Model is an example of a "Stepping Stone" model.

\subsection{The Primary Model}

The Primary Model is a system of three coupled ODEs, which, in autonomous form, are suggested in Nevai and Van Gorder (2012) as

$$
\begin{aligned}
\frac{\mathrm{d} x}{\mathrm{~d} t} & =r x\left(1-\frac{x}{k}\right)-\theta\left(\frac{x}{x+s+h}\right) y, \\
\frac{\mathrm{d} s}{\mathrm{~d} t} & =i-\gamma s-\psi\left(\frac{s}{x+s+h}\right) y, \\
\frac{\mathrm{d} y}{\mathrm{~d} t} & =\left(\frac{\epsilon \theta x+\eta \psi s}{x+s+h}\right) y-\delta y,
\end{aligned}
$$

where $x(t), s(t)$, and $y(t)$ are the population sizes of the prey, subsidy, and predator respectively. The first term in Equation (2.1) is the production of the prey by logistic growth, where $r$ is the intrinsic growth rate of the prey, and $k$ is the carrying capacity of the prey. The second term in Equation (2.1) describes the rate at which the prey is consumed by the predator, where $\theta$ is the maximum rate at which the predators exploits the prey, and $h$ is the half-saturation parameter.

In Equation (2.2), $i$ is the rate at which the subsidy is created, and $\gamma$ is the rate at which it decays. The final term in Equation (2.2) describes the rate at which the subsidy is consumed by the predator, where $\psi$ is the maximum rate at which the predator can exploit the subsidy. The first term in Equation (2.3) describes how the predator benefits from its food sources, where $\epsilon$ and $\eta$ are conversion factors. The final term in Equation (2.3) describes the death of the predator, where $\delta$ is the mortality rate.

Nevai and Van Gorder (2012) found that the autonomous system (2.1)-(2.3) permitted coexistence equilibira, extinction equlibria, and limit cycles for certain appropriate parameter regimes. No chaotic dynamics were observed for the autonomous primary model of Nevai and Van Gorder (2012). In Section 2.3, we extend the analysis in Nevai and Van Gorder (2012) by considering the effect of time-dependent parameters $r(t), k(t), i(t), \gamma(t)$, and $\delta(t)$. The form of these parameters will be motivated by seasonality in the the Arctic ecosystem of the arctic foxes, lemmings, and seal carrion.

\subsection{The n-Patch Model}

The second model considered is the $n$-Patch Model. A patch is a bounded region of space which is large enough such that each individual prey does not venture out of its patch. The patches are disconnected. Therefore, the population of prey and of subsidy within each patch is not directly influenced by the populations in other patches. The predator population can move between patches.

In this model, there exist $n$ patches, $\left\{\mathcal{P}^{(1)}, \mathcal{P}^{(2)}, \ldots, \mathcal{P}^{(n)}\right\}$. Let $x^{(j)}(t), s^{(j)}(t)$, and $y^{(j)}(t)$ represent the population sizes of the prey, subsidy and predator respectively in patch $\mathcal{P}^{(j)}$ for $j=$ $1, \ldots, n$. We generalise the two-patch model in Nevai and Van 
Gorder (2012), and present the $n$-Patch Model. It is a $3 n^{\text {th }}$ order system given by the system of equations

$$
\begin{array}{r}
\frac{\mathrm{d} x^{(j)}}{\mathrm{d} t}=r^{(j)} x^{(j)}\left(1-\frac{x^{(j)}}{k^{(j)}}\right)-\theta\left(\frac{x^{(j)}}{x^{(j)}+s^{(j)}+h}\right) y^{(j)}, \\
\frac{\mathrm{d} s^{(j)}}{\mathrm{d} t}=i^{(j)}-\gamma^{(j)} s^{(j)}-\psi\left(\frac{s^{(j)}}{x^{(j)}+s^{(j)}+h}\right) y^{(j)}, \\
\frac{\mathrm{d} y^{(j)}}{\mathrm{d} t}=\left(\frac{\epsilon \theta x^{(j)}+\eta \psi s^{(j)}}{x^{(j)}+s^{(j)}+h}\right) y^{(j)}-\delta^{(j)} y^{(j)} \\
+\sum_{\ell=1}^{n}\left(\lambda^{(\ell, j)} \alpha^{(\ell, j)} y^{(\ell)}-\alpha^{(j, \ell)} y^{(j)}\right),
\end{array}
$$

where, in the $j^{\text {th }}$ patch, $r^{(j)}(t)$ is the prey intrinsic growth rate; $k^{(j)}(t)$ is the prey carrying capacity; $i^{(j)}(t)$ is the rate at which the the subsidy is created; $\gamma^{(j)}(t)$ is the rate at which the subsidy decays; and $\delta^{(j)}(t)$ is the predator mortality rate. The rate at which the predator travels from $\mathcal{P}^{(j)}$ to $\mathcal{P}^{(\ell)}$ is represented by $\alpha^{(j, \ell)}(t)$. Note that it does not makes sense to consider predator migration from a patch to itself and therefore we can set $\alpha^{(j, j)}(t) \equiv 0$ for all $j$. Finally, $\lambda^{(j, \ell)}$ describes the proportion of the predator which survive inter-patch migration from $\mathcal{P}^{(j)}$ to $\mathcal{P}^{(\ell)}$. This is implicitly equal to unity in (Nevai and Van Gorder, 2012) which does not consider mortality rates due to migration.

The parameters $\theta, \psi, e, \epsilon$, and $\eta$ describe the interactions between the predator, the prey, and the subsidy, which is homogeneous in all patches. Therefore we do not consider those parameters to depend on the patch.

\subsection{Choice Of Time-Dependent Parameters}

Without loss of generality, we consider $t=0$ to occur in the middle of winter, and let $\tau$ denote the length of a year in the natural timescale of the model.

The choice for the functions for $i(t)$ and $i^{(j)}(t)$ is motivated by the seasonal diet of the polar bears in the gulf of Boothia (Thiemann et al., 2008). In the spring-summer when the ice recedes, the polar bears eat few seals due to the availability of aquatic migratory species such as the the beluga whale and the narwhal. Consequently there are fewer seal carrion for the arctic fox than during the autumn-winter period.

Therefore, $i(t)$ and $i^{(j)}(t)$ should be one-year periodic. The simplest analytic periodic functions are arguably sinusoidal and therefore we consider

$$
\begin{aligned}
i(t) & =i_{0}\left(1+i_{1} \cos \left(\frac{2 \pi t}{\tau}\right)\right), \\
i^{(j)}(t) & =i_{0}^{(j)}\left(1+i_{1}^{(j)} \cos \left(\frac{2 \pi t}{\tau}\right)\right) .
\end{aligned}
$$

Here, $i_{0}$ and $i_{0}^{(j)}$ are the average annual rates of subsidy input for the Primary and $n$-Patch Model; and $i_{1}$ and $i_{1}^{(j)}$ are the amplitude of oscillations about the mean for the two systems respectfully.

There are many factors that contribute to the rate of decay of animal carrion, including the mass and location of the corpse; and the chemistry of the soil (Meyer et al., 2013). However, one of the most important factors is the temperature (Meyer et al., 2013; Talent, 2012). Despite this, very few studies have investigated the effect of seasonality on carrion decomposition. One example is a study of swine in which corpses decomposed much faster in the summer than in the winter (Meyer et al., 2013). In this paper we propose the form for the decay rate of the seal carrion to be

$$
\begin{aligned}
\gamma(t) & =\gamma_{0}\left(e^{\gamma_{1} T(t)}-1\right), \\
\gamma^{(j)}(t) & =\gamma_{0}^{(j)}\left(e^{\gamma_{1}^{(j)} T^{(j)}(t)}-1\right),
\end{aligned}
$$

where $T(t)$ and $T^{(j)}(t)$ are the absolute temperatures at time $t$; $\gamma_{0}$ and $\gamma_{0}^{(j)}$ are reference decay rates; and $\gamma_{1}$ and $\gamma_{1}^{(j)}$ are temperature scaling parameter.

The functional form describing the temperature, $T(t)$ and $T^{(j)}(t)$, suggested in Nevai and Van Gorder (2012) but translated into time, are

$$
\begin{aligned}
T(t) & =T_{0}+m t-A_{0} \cos \left(\frac{2 \pi t}{\tau}\right), \quad t=\frac{\lfloor n\rfloor}{365}, \\
T^{(j)}(t) & =T_{0}^{(j)}+m t-A_{0}^{(j)} \cos \left(\frac{2 \pi t}{\tau}\right),
\end{aligned}
$$

where $T_{0}$ and $T_{0}^{(j)}$ are reference temperatures which we set to be $T_{0}=T_{0}^{(j)}=258 \mathrm{~K}$ (Danish Meteorological Institute, 2015); $A_{0}$ and $A_{0}^{(j)}$ are the amplitudes of the yearly fluctuations in the temperature and are strictly positive, and we set them to equal $A_{0}=A_{0}^{(j)}=16 K$ (Danish Meteorological Institute, 2015); $n$ is the amount of time which has passed in days; and $m$ describes long term effects of the change in the weather and global warming. In this paper, we set $m=0 K$ year $^{-1}$ as we are investigating seasonal effects, however a typical value is $m=0.006 \mathrm{~K}_{\text {year }^{-1}}$ (Root et al., 2003).

The temperature functions given in Equations (2.11)-(2.12) predict that the temperature grows without bound. In the present paper, we shall simply be interested in the periodic part, due to seasonal variations, and hence we set $m=0$. We shall therefore use

$$
\begin{aligned}
T(t) & =T_{0}-A_{0} \cos \left(\frac{2 \pi t}{\tau}\right), \\
T^{(j)}(t) & =T_{0}^{(j)}-A_{0}^{(j)} \cos \left(\frac{2 \pi t}{\tau}\right),
\end{aligned}
$$

which are now bounded. With this, we now suggest forms for the decay rate of the subsidy

$$
\begin{aligned}
\gamma(t) & =\frac{\gamma_{0}}{e^{\gamma_{1} T_{0}} I_{0}\left(\gamma_{1} A_{0}\right)-1}\left(e^{\gamma_{1} T(t)}-1\right), \\
\gamma^{(j)}(t) & =\frac{\gamma_{0}^{(j)}}{e^{\gamma_{1}^{(j)} T_{0}^{(j)} I_{0}\left(\gamma_{1}^{(j)} A_{0}^{(j)}\right)-1}\left(e^{\gamma_{1}^{(j)} T^{(j)}(t)}-1\right),}
\end{aligned}
$$

where $I_{0}(\cdot)$ is the modified Bessel function of the first kind which appears due to an integral of an exponential of a cosine function arising when we normalize the functions $\gamma(t)$ and $\gamma^{(j)}(t)$ over a period. The advantage of this form is that the reference decay rates, $\gamma_{0}$ and $\gamma_{0}^{(j)}$, now describes the average annual decay rates, thanks to this normalization. Note that as $\gamma_{1} \rightarrow 0$, 
we have $\gamma(t) \rightarrow \gamma_{0}$ and so we can use this limit to describe the time-independent parameter. Similarly, as $\gamma_{1}^{(j)} \rightarrow 0$, we have $\gamma^{(j)}(t) \rightarrow \gamma_{0}^{(j)}$.

There are two main causes of arctic fox mortality. The first is due to seasonality. During the winter, food is generally scarcer and therefore starvation increases death rates in this period (Prestrud, 1991). The second is due to migration. This occurs every 3-4 years (Wrigley and Hatch, 1976). Dangers during migration include the lack of food; the risk of disease - three quarters of the population are affected by rabies; and some foxes are trapped for use in the fur industry (Wrigley and Hatch, 1976). Therefore, the function for the predator mortality rate in the Primary Model is

$$
\delta(t)=\delta_{0}\left(1+\delta_{1} \cos \left(\frac{2 \pi t}{\tau}\right)\right)\left(1+\delta_{2} \cos \left(\frac{2 \pi t}{\tau_{2}}\right)\right),
$$

where $\tau_{2} \neq \tau$ to account for the difference between seasonality and migration time frames. When performing numerical simulations, we shall take $\tau_{2}=4 \tau$ in agreement with the findings of Wrigley and Hatch (1976). The average mortality rate is given by $\delta_{0}$ and the importance of the seasonal, and quadrennial migration effects on the mortality rate are given by $\delta_{1}$ and $\delta_{2}$ respectfully.

In the $n$-Patch Model, we absorb the effects of migration on predator mortality into the parameter $\lambda^{(j, \ell)}$, and describe the migration rate by the function $\alpha^{(j, \ell)}(t)$. Therefore, for the $n$-Patch Model we use

$$
\begin{aligned}
\delta^{(j)}(t) & =\delta_{0}^{(j)}\left(1+\delta_{1}^{(j)} \cos \left(\frac{2 \pi t}{\tau}\right)\right), \\
\alpha^{(j, \ell)}(t) & = \begin{cases}\alpha_{0}^{(j, \ell)}\left(1+\alpha_{1}^{(j, \ell)} \cos \left(\frac{2 \pi t}{\tau_{2}}\right)\right), & j \neq \ell, \\
0, & j=\ell,\end{cases}
\end{aligned}
$$

where $\delta_{0}^{(j)}$ is the average predator mortality rate in $\mathcal{P}^{(j)}, \delta_{1}^{(j)}$ is the amplitude of oscillations of predator migration, $\alpha_{0}^{(j, \ell)}$ is the average rate of migration from $\mathcal{P}^{(j)}$ to $\mathcal{P}^{(\ell)}$, and $\alpha_{1}^{(j, \ell)}$ is the amplitude of oscillations about the mean. When performing numerical simulations, we shall again take $\tau_{2}=4 \tau$, which is motivated by the findings of Wrigley and Hatch (1976).

Finally, Turchin and Hanski (1997); Rinaldi et al. (1993) suggest that seasonal effects on the growth rate of the lemmings may mean that $r(t)$ and $k(t)$, and $r^{(j)}(t)$ and $k^{(j)}(t)$, vary sinusoidally in a coupled manner. The form suggested is

$$
\begin{gathered}
r(t)=r_{0}\left(1-r_{1} \cos \left(\frac{2 \pi t}{\tau}\right)\right), k(t)=k_{0}\left(1-k_{1} \cos \left(\frac{2 \pi t}{\tau}\right)\right), \\
r^{(j)}(t)=r_{0}^{(j)}\left(1-r_{1}^{(j)} \cos \left(\frac{2 \pi t}{\tau}\right)\right), k^{(j)}(t)=k_{0}^{(j)}\left(1-k_{1}^{(j)} \cos \left(\frac{2 \pi t}{\tau}\right)\right) .
\end{gathered}
$$

Here, $r_{0}$ and $r_{0}^{(j)}$ are the average values of the prey intrinsic growth rate, and $k_{0}$ and $k_{0}^{(j)}$ are the average values of the prey carrying capacity respectfully. Moreover, $r_{1}, r_{1}^{(j)}, k_{1}$, and $k_{1}^{(j)}$ describe the oscillations of these quantities about the mean. The coupling given in Turchin and Hanski (1997) implies that $r_{1}=$ $k_{1}$ and $r_{1}^{(j)}=k_{1}^{(j)}$, so that $\frac{r(t)}{k(t)}$ and $\frac{r^{(j)}(t)}{k^{(j)}(t)}$ are constants. We use this fact in the rest of the paper. We also require that the parameters $i_{0}, r_{0}, k_{0}, \delta_{0}, \alpha_{0}, \gamma_{0}, \gamma_{1}$ are positive, while $i_{1}, r_{1}, k_{1}, \delta_{1}, \delta_{2}, \alpha_{1}, \lambda$ are between zero and unity, for all superscripts.

\section{The Primary Model}

This section considers the Primary Model given in Equations (2.1)-(2.3) with the time-dependent functions given in Section 2 . We begin by analysing the equations analytically. In Section 3.1, we prove that the dynamics of the system are bounded above and remain non-negative, and are hence biologically feasible. Then in Section 3.2 we examine the effect that the subsidy, $s(t)$, has on the population dynamics of the predator, $y(t)$, and the prey, $x(t)$. In Section 3.3, we numerically analyse the sensitivity of the solutions to the parameters.

\subsection{Bounded Dynamics}

The population sizes of the predator, prey, and subsidy must remain non-negative in order to be biologically feasible. Moreover, we expect features of the ecological system such as competition and limited resources to provide a natural upper bound for the populations sizes. The following theorem shows that our equations are consistent with these observations.

Theorem 3.1. Consider the predator-prey-subsidy Primary Model given by Equations (2.1)-(2.3). Let all the parameters and the initial conditions be strictly positive. Suppose that $r(t)$, $k(t), i(t)$, and $\gamma(t)$ are bounded above. Suppose further that $i(t)$, $\gamma(t)$, and $\delta(t)$ are bounded below away from zero. Then $x(t)$, $s(t)$, and $y(t)$ are bounded below by zero, and have theoretical maximum values. Moreover, $s(t)$ is bounded below away from zero.

Proof. See Lemmas A.1 - A.3 in Appendix A.

\subsection{Effect Of Subsidy On Predator And Prey}

In this section, we examine the effect of introducing a subsidy into the predator-prey system. We do this to demonstrate the importance of considering the subsidy when the subsidy is sufficiently influential in the system.

Figure 1 shows the effect of increasing the rate of input of the subsidy, $i_{0}$, on the population size of the predator and prey for two different systems. Where the graph is single-valued, an equilibrium is approached and is displayed. Where the graph is multi-valued, we display the minimum and maximum values of the limit cycle generated. When the unperturbed system has a stable equilibrium, the system approaches an equilibrium when no subsidy is present. As $i_{0}$ increases from zero, there is more available subsidy and consequently more food available for the predator. The predator population size increases. Consequently, the prey population size decreases as there is a higher risk of predation. Interestingly, as soon as the rate of subsidy input is sufficiently large to cause prey extinction, the predator population size at equilibrium increases at a larger rate as the subsidy input rate increases. When the unperturbed system has 
a limit cycle, increasing $i_{0}$ has the effect of stabilising the dynamics. When the value of $i_{0}$ is sufficiently large, the prey is removed from the system, and once again there is an increased sensitivity of the equilibrium value of the predator to the input rate of the subsidy.

\subsection{Numerical Simulations}

In this section, we investigate the effect of the timedependent parameters on the predator-prey-subsidy system using numerical simulations. All the numerical simulations have been carried out using MATLAB's ode45 solver (MATLAB, 2014) which uses Runge-Kutta $(4,5)$. We lowered the relative tolerance of the solver to $10^{-12}$ to reduce the risk of numerical error influencing the dynamics, especially prevalent for small $\tau$. We consider two cases, determined by the values of their parameters in Figure 2 (Nevai and Van Gorder, 2012).

\subsubsection{Effect Of Oscillations On Equilibria}

We examine the effect of introducing a time-dependent parameter to the autonomous model which predicted the positive equilibrium present in Figure 2, in Figure 3. Figure 3(a) introduces time-dependent prey growth rate, $r(t)$, and prey carrying capacity, $k(t)$; Figure 3(b)-(c) consider time-dependent predator mortality rate, $\delta(t)$; Figure $3(\mathrm{~d})$ examines the effect of timedependent subsidy input rate, $i(t)$; and Figure 3(e) investigates time-dependent subsidy decay rate, $\gamma(t)$.
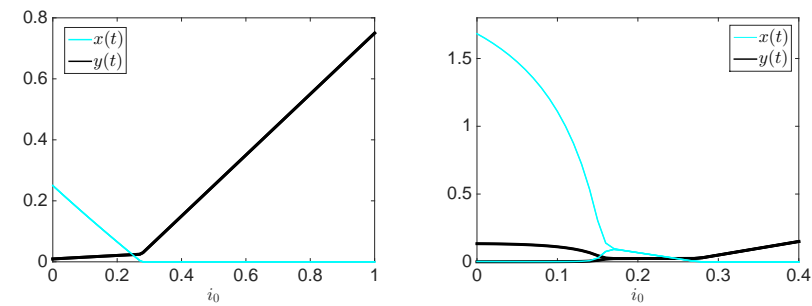

Figure 1: The effect of the subsidy input rate, $i_{0}$, on the stable equilibria (left) and limit cycles (right) of the autonomous system. The maximum and minimum values of the stable dynamics have been plotted. The parameters used are $r_{0}=$ $0.1, r_{1}=0, \theta=5, h=1, i_{1}=0, \gamma_{0}=1, \gamma_{1}=0, \psi=5, \epsilon=0.1, \eta=0.1$, $\delta_{0}=0.1, \delta_{1}=0$, and $\delta_{2}=0$. The initial conditions are $x(0)=0.2, s(0)=0$, and $y(0)=0.1$. We take $k_{0}^{(j)}=0.4$ (left) and $k_{0}^{(j)}=2.4$ (right). The value of $i_{0}$ is the dependent variable here.
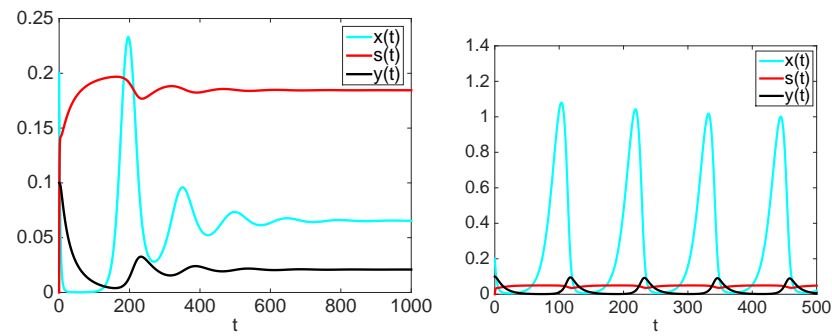

Figure 2: The dynamics of the predator-prey-subsidy system when all parameters are constants. The parameters are $x(0)=0.2, s(0)=0, y(0)=0.1, \theta=5$, $h=1, \psi=5, \epsilon=0.1, \eta=0.1, r_{0}=0.1, r_{1}=0, i_{1}=0, \gamma_{0}=1, \gamma_{1}=0$, $\delta_{0}=0.1, \delta_{1}=0$, and $\delta_{2}=0$. The left panel has $k_{0}=0.4$ and $i_{0}=0.2$ which give a positive equilibrium; the right panel has $k_{0}=2$ and $i_{0}=0.05$ which give a limit cycle.

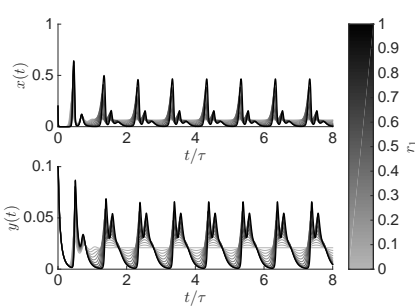

(a) Varying $r_{1}$.

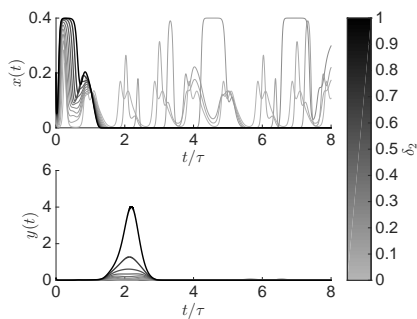

(c) Varying $\delta_{2}$.

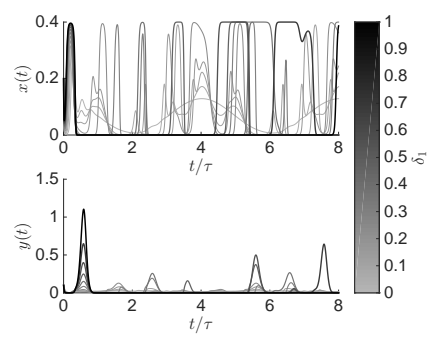

(b) Varying $\delta_{1}$.

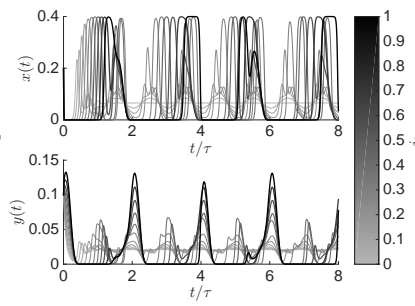

(d) Varying $i_{1}$.

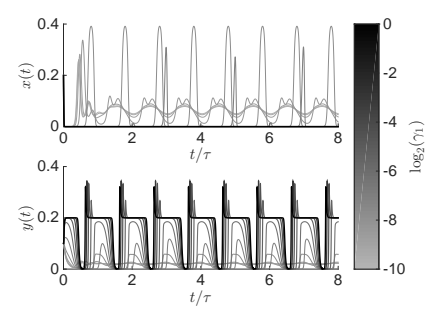

(e) Varying $\gamma_{1}$.

Figure 3: Effect of the time-dependent parameters' amplitude of oscillations on the dynamics of the prey, $x(t)$, and the predator, $y(t)$, in the case of a positive equilibrium in the unperturbed system shown in Figure 2. In (a), $r(t)$ is timedependent and we examine the effect of $r_{1}$. In (b)-(c), $\delta(t)$ is time dependent and we examine the effect varying of $\delta_{1}$ and $\delta_{2}$ respectfully whist keeping the other one fixed at a value of 0.5. In Figure 3(d), $i(t)$ is time-dependent and we examine the effect of varying $i_{1}$. Finally, in (e), $\gamma(t)$ is time-dependent and we examine the effect of varying $\gamma_{1}$. We have used $\tau=500$ throughout.

We find that the solution to the equations evolves smoothly as $r_{1}$ is varied in the case where $r(t)$ is non-constant. It has the effect of introducing limit cycles with period-2 oscillations - twice the period of $r(t)$. Neither the predator nor the prey is driven to extinction. This contrasts the results found when varying $\gamma(t)$ where for large enough values of $\gamma_{1}$, the prey is driven to extinction. We still find that the solution when $\gamma(t)$ is non-constant evolves smoothly as $\gamma_{1}$ is varied, and that the period of the oscillations is biennial.

When considering time-dependent predator mortality rate, $\delta(t)$, we observe that large values of $\delta_{1}$ or $\delta_{2}$ are able to drive both the predator and prey to extinction. This occurs because the amount of predator becomes very large, driving the prey to extinction and then the ecosystem is unable to sustain the predator which subsequently becomes extinct as well. This dynamics is known as boom and bust dynamics (Barnes, 2002; Stott et al., 2010; Kingsford et al., 1999; Lee, 1974; Dickman et al., 2010). For small values of $\delta_{1}$ or $\delta_{2}$ where the predator and prey do not go extinct, the solutions do not evolve smoothly as the parameters are varied.

Finally, we see that time-dependent $i(t)$ creates limit cycles in the dynamics. The limit cycles have period- 2 oscillations for 
large values of $i_{1}$. As $i_{1}$ is increased, the peak of the solution for the prey, $x(t)$, moves smoothly rightwards. The peak for the predator, $y(t)$, moves to the right, but not by the same magnitude.

\subsubsection{Effect Of Oscillations On Limit Cycles}

Next, we investigate the effect of introducing time-dependent parameters to the autonomous system which predicted limit cycles. We first wish to explore the interaction between the oscillations of the limit cycles and the oscillations of the parameters. The period of the limit cycles of the autonomous system in the natural timescale of the system is 110 units of time. We conduct the investigation by allowing all possible parameters to be time-dependent and choose the time-length of a year in the natural timescale of the system, $\tau$, to be very small in comparison $(\tau=1)$ in Figure 4(a); of a similar magnitude $(\tau=500)$ in Figure 4(b); and very large in comparison $(\tau=3000)$ in Figure 4(c). We find that for small values of $\tau$, the natural limit cycles dominate; for large values of $\tau$, the oscillations of the parameters dominate; and for comparable values of $\tau$, there is interference between the two oscillations which results in aperiodic behaviours.

We research the effect of considering time-dependent parameters rather than constant parameters shown in the right panel of Figure 2, in Figure 5. In Figure 5(a), $r(t)$ and $k(t)$ are timedependent; in Figure 5(b)-(c), $\delta(t)$ is time-dependent; in Figure 5(d), $i(t)$ depends on time; and in Figure 5(e), $\gamma(t)$ depends on time.

We find that $i(t)$ has very little effect on the dynamics, but does break the regularity of the limit cycles. We observe the boom and bust dynamics once again for large values of $\delta_{1}$ or $\delta_{2}$ when $\delta(t)$ is depends on time. For small values of $\delta_{1}$ and $\delta_{2}$, the dynamics is very irregular. When $r(t)$ is time dependent, both the prey and the predator survive, but the dynamics is aperiodic. Finally, for large values of $\gamma_{1}$ in Figure 5(e), we find that the prey goes extinct, and the predator follows regular limit cycles. For smaller values of $\gamma_{1}$, both prey and predator survive and undergo oscillations. As we shall show later, some periodic perturbations of stable limit cycles by the input of subsidy, $i(t)$, can push these stable limit cycles into quasi-periodic dynamics on invariant tori.

The dynamics in Figures 5(a)-(d) look like they may exhibit chaos. This is explored further in Section 5.

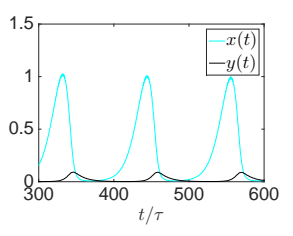

(a) $\tau=1$

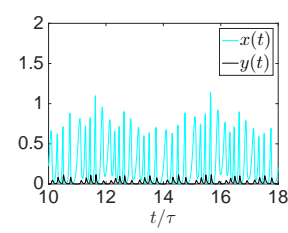

(b) $\tau=500$

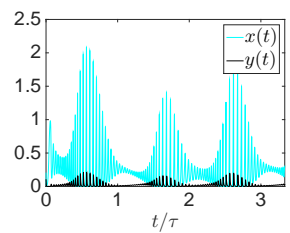

(c) $\tau=3000$
Figure 4: The effect of $\tau$ on the limit cycles produced in Figure 2 when time dependent parameters are introduced. We have $r_{1}=0.5, i_{1}=0.1, \gamma_{1}=0.2$, $\delta_{1}=0.2$, and $\delta_{2}=0.2$. The value of $\tau$ is given under each figure.

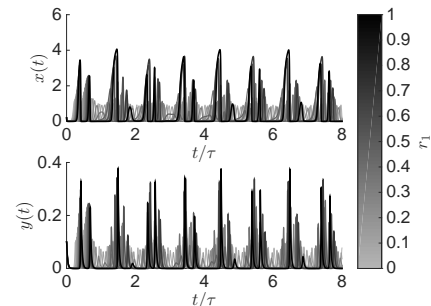

(a) Varying $r_{1}$.

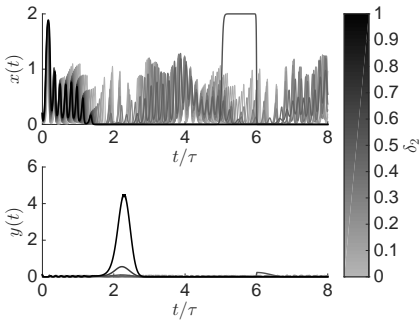

(c) Varying $\delta_{2}$.

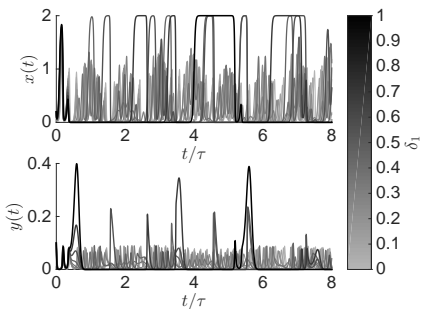

(b) Varying $\delta_{1}$.

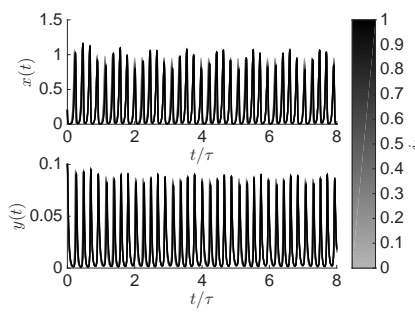

(d) Varying $i_{1}$.

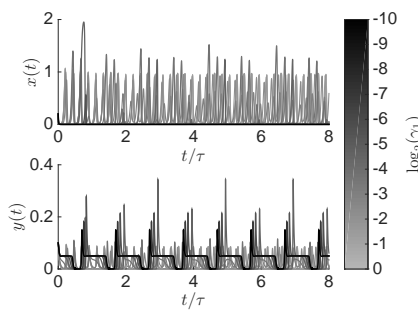

(e) Varying $\gamma_{1}$

Figure 5: Examination of the effect of the amplitude of oscillations of the timedependent parameters on the dynamics of the prey $(x(t))$ and the predator $(y(t))$ in the case where the unperturbed system gives the limit cycle shown in Figure 2. In (a), $r(t)$ is time dependent and we examine the effect of $r_{1}$. In (b)-(c), $\delta(t)$ is time dependent and we examine the effect varying of $\delta_{1}$ and $\delta_{2}$ respectfully whist keeping the other one fixed at a value of 0.5 . In (d), $i(t)$ is time-dependent and we examine the effect of varying $i_{1}$. Finally, in (e), $\gamma(t)$ is time-dependent and we examine the effect of varying $\gamma_{1}$. We have $\tau=500$ throughout.

\section{The $n$-Patch Model}

This section analyses Equations (2.4)-(2.6) which describe the $n$-Patch Model for the predator-prey-subsidy system, together with the parameters in Section 2. We begin in Section 4.1 where we prove that the dynamics of the system is bounded above, and bounded below by zero. Then, we show in Section 4.2 that if the patches are homogeneous and the initial conditions are identical, then we can analyse the system using the Primary Model. Next, in Section 4.3, we examine limit cycles which are induced by the predator mortality rate during migration. In Section 4.4, we examine the effect of introducing subsidy to a predator-prey system. Next, we consider a three-patch model to describe migration between two spatially separated patches, where the third patch describes predators in the migratory state, in Section 4.5. Finally, in Section 4.6, we examine the effect of the amplitude of oscillations of the predator migration rate on the dynamics of two systems. The first approaches an equilibrium in the autonomous system and the second generates a limit cycle. 


\subsection{Bounded Dynamics}

In a similar manner to the Primary Model, we wish to determine whether the dynamics for the $n$-Patch Model are bounded above, and bounded below by zero.

Theorem 4.1 (Bounded Dynamics). Consider the predatorprey-subsidy n-Patch Model given by Equations (2.4)-(2.6). Let all the parameters and initial conditions be non-negative. Suppose that $r^{(j)}(t), k^{(j)}(t)$, and $i^{(j)}(t)$ are bounded above. Suppose further that $\delta^{(j)}(t)$, and $\alpha^{(j, \ell)}(t)$ are bounded below away from zero. Then the predator, prey, and subsidy population sizes are bounded above, and bounded below by zero.

Proof. See Lemmas B.1-B.2 in Appendix B.

\subsection{Homogeneous Patches}

Consider the $n$-Patch Model with homogeneous patches and identical initial conditions. We write $r^{(j)}(t)=r(t), k^{(j)}(t)=k(t)$, $i^{(j)}(t)=i(t), \gamma^{(j)}(t)=\gamma(t), \delta^{(j)}(t)=\delta(t), \alpha^{(j, \ell)}(t)=\alpha(t)$, and $\lambda^{(j, \ell)}=\lambda$ for all $j$ and $\ell$ with $j \neq \ell$, due to the homogeneity. Because of the indistinguishability of the patches, the dynamics in each patch must be the same. Therefore, we let $x(t), s(t)$, and $y(t)$ represent the prey, subsidy, and predator population sizes in any patch. Equations (2.4-2.6) can then be written as

$$
\begin{aligned}
\frac{\mathrm{d} x}{\mathrm{~d} t} & =r x\left(1-\frac{x}{k}\right)-\theta\left(\frac{x}{x+s+h}\right) y, \\
\frac{\mathrm{d} s}{\mathrm{~d} t} & =i-\gamma s-\psi\left(\frac{s}{x+s+h}\right) y, \\
\frac{\mathrm{d} y}{\mathrm{~d} t} & =\left(\frac{\epsilon \theta x+\eta \psi s}{x+s+h}\right) y-(\delta+\alpha(1-\lambda)) y .
\end{aligned}
$$

These equations are identical to those in the Primary Model, where the annual oscillations of $\delta(t)$ and the quadrennial oscillations of $\alpha(t)$ combine to form a function with the same form as that of the predator mortality rate given in the Primary Model.

Next we wish to find the equations satisfied by the total populations. We let $X(t), S(t)$, and $Y(t)$ represent the total population sizes of the prey, subsidy and predator in all the patches. Note that due to the parity between all patches, we could equivalently define $X(t)=n x(t), S(t)=n s(t)$, and $Y(t)=n y(t)$. These functions satisfy

$$
\begin{aligned}
\frac{\mathrm{d} X}{\mathrm{~d} t} & =r X\left(1-\frac{X}{K}\right)-\theta\left(\frac{X}{X+S+H}\right) Y, \\
\frac{\mathrm{d} S}{\mathrm{~d} t} & =I-\gamma S-\psi\left(\frac{S}{X+S+H}\right) Y, \\
\frac{\mathrm{d} Y}{\mathrm{~d} t} & =\left(\frac{\epsilon \theta X+\eta \psi S}{X+S+H}\right) Y-(\delta+\alpha(1-\lambda)) Y,
\end{aligned}
$$

where $K(t)=n k(t), H=n h$, and $I(t)=n i(t)$ are global parameters. Hence, the dynamics of the total populations can also be modelled by the Primary Model, but with three scaled parameters. Subsidy is inputted into each patch at the same rate which is why the total amount of subsidy of the system, $I(t)$, is proportional to the number of patches. Similarly, each patch can support the same amount of prey and therefore the amount of prey the system can support, $K(t)$, is proportional to the number of patches. The proportion of prey and subsidy in the entire system immediately available to each predator is inversely proportional to the number of patches, which biologically justifies the form of the new half-saturation parameter, $H$. The parameters $r, \theta, \gamma, \psi, \epsilon, \eta, \delta, \alpha$, and $\lambda$ are not proportional to the number of patches.

\subsection{Migration-Induced Limit Cycles}

We now look at the effect that the proportion of migration survival, $\lambda^{(j, \ell)}$, has on the dynamics. The particular example considered is a two-patch system in which the first patch is subsidy-free, and the second patch is prey-free. This is achieved by setting $s^{(1)}(0)=0, i^{(1)}(t) \equiv 0$, and $x^{(2)}(0)=0$. We let $\lambda^{(1,2)}=\lambda^{(2,1)}=\lambda$.

Figure 6 shows the maximum and minimum population sizes attained by the prey, $x(t)$, and the predator, $y(t)$, in the autonomous system once transient behaviours have concluded, as $\lambda$ is varied. Where the graphs are single-valued, an equilibrium is reached, whilst where the graphs are multi-valued, a limit cycle is induced. In this example, there is a region in $\lambda$-parameter space in which limit cycles exist, and outside of this region the dynamics approaches an equilibrium

It is natural to wonder what happens when the migration terms go out of phase. This would correspond to predator preference for one region during one time interval, and preference for a different region over another time interval. To consider such a situation in the two-patch framework, we take have used that

$$
\alpha^{(2,1)}(t)=\alpha_{0}\left(1+\alpha_{1} \cos \left(\frac{\pi(t+\varphi)}{2 \tau}\right)\right)
$$

while $\alpha^{(1,2)}(t)$ is as given in (2.18). Here $\varphi \in[0,4 \tau)$ is the parameter which determines the degree to which the two migration functions are out of sync. We plot the behavior of the populations as a function of the phase parameter $\varphi$ in Figure 7. Notice that being out of phase seems to have an overall positive effect on the predator, and does not effect the average value for the prey. Most interestingly, perhaps, is the apparent symmetry between the predator and subsidy. This highlights the difference between a food source which is self replicating (prey), and a food source which is provided at a rate independent of its current size (subsidy), showing a much stronger correlation between predator and subsidy than predator and prey. It therefore

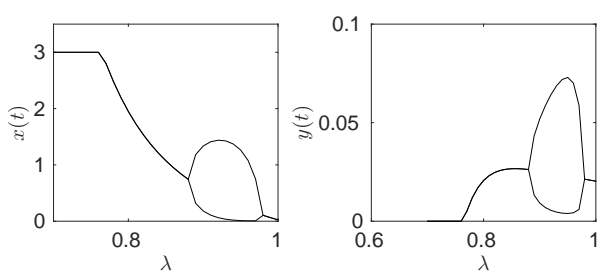

Figure 6: Bifurcation diagram of $x(t)$ and $y(t)$ as the proportion of predator migration survival, $\lambda$, is varied. Graphs show the maximum and minimum values attained once transient behaviour is over. The parameters used are $r_{0}^{(j)}=$ $0.1, r_{1}^{(j)}=0, k_{0}^{(j)}=3, \theta=5, h=1, i_{0}^{(1)}=0, i_{0}^{(2)}=0.6, i_{1}^{(j)}=0, \gamma_{0}^{(j)}=1$, $\gamma_{1}^{(j)}=0, \psi=5, \epsilon=0.1, \eta=0.1, \delta_{0}^{(j)}=0.1, \delta_{1}^{(j)}=0, \alpha_{0}^{(j, \ell)}=0.8, \alpha_{1}^{(j, \ell)}=0$, and $\tau=500$. The initial conditions are $x^{(1)}(0)=0.2, x^{(2)}(0)=0, s^{(1)}(0)=0$, $s^{(2)}(0)=0, y^{(1)}(0)=0.1$, and $y^{(2)}(0)=0$. 
goes to show the importance of including the resource subsidy in the model.

\subsection{Effect Of Subsidy On Predator And Prey}

We repeat the analysis in Section 3.2 to examine the effect of the input rate of the subsidy on the autonomous $n$-Patch Model. We examine a two-patch model for which the first patch is subsidy-free and the second patch is prey-free in Figure 8. Similar observations can be made as in Section 3.2, including bifurcation of limit cycles into equilibria, and the increased sensitivity of the predator to the rate of subsidy input once the prey is extinct.

\subsection{Three-Patch Model}

We have accounted for predator mortality rates due to migration in two unique ways. In Section 3 , it was incorporated into $\delta(t)$. Then, in this section, the parameter $\lambda^{(j, \ell)}$ described the proportion of the predators which survive inter-patch migration.

A third approach is to use a three-patch model, where patches $\mathcal{P}^{(1)}$ and $\mathcal{P}^{(3)}$ contain prey and subsidy, but patch $\mathcal{P}^{(2)}$ does not. In order for the predator to travel between $\mathcal{P}^{(1)}$ and $\mathcal{P}^{(3)}$, it must travel through $\mathcal{P}^{(2)}$ where its mortality rate, $\delta^{(2)}(t)$, is larger than

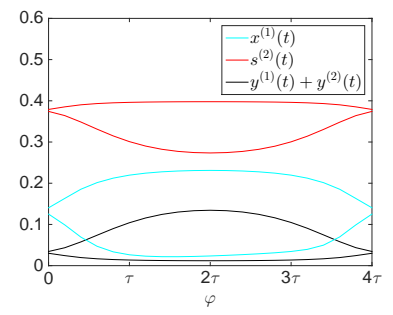

Figure 7: Bifurcation diagram of $x(t)$ and $y(t)$ as the relative phase $\varphi$ of the two migration functions, $\alpha^{(1,2)}(t)$ and $\alpha^{(2,1)}(t)$, is varied. Graphs show the maximum and minimum values attained once transient behaviour is over. The parameters used are $r_{0}^{(1)}=0.1, r_{0}^{(2)}=0, r_{1}^{(j)}=0, k_{0}^{(j)}=0.4, \theta=5, h=1, i_{0}^{(1)}=0$, $i_{0}^{(2)}=0.4, i_{1}^{(j)}=0, \gamma_{0}^{(j)}=0.2, \gamma_{1}^{(j)}=0, \psi=5, \epsilon=0.1, \eta=0.1, \delta_{0}^{(j)}=0.1$, $\delta_{1}^{(j)}=0, \alpha_{0}^{(j, \ell)}=0.8, \alpha_{1}^{(j, \ell)}=0.75, \lambda^{(j, \ell)}=1, \tau=500, x^{(1)}(0)=0.2, x^{(2)}(0)=0$, $s^{(1)}(0)=0, s^{(2)}(0)=0, y^{(1)}(0)=0.1, y^{(2)}(0)=0$. (a) Stable Equilibrium

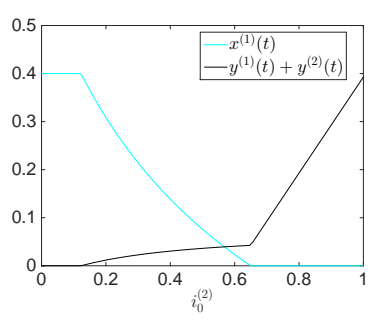

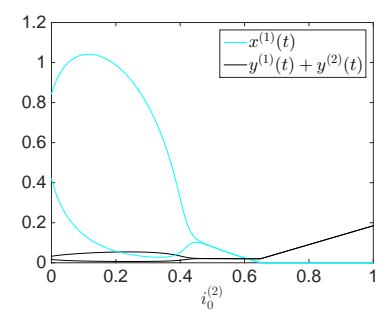

(b) Limit Cycles
Figure 8: The effect of the subsidy input rate, $i_{0}^{(2)}$, on the stable equilibria (left) and limit cycles (right) of the autonomous two-patch system. The maximum and minimum values of the stable dynamics have been plotted. The parameters used are $r_{0}^{(j)}=0.1, r_{1}^{(j)}=0, k_{0}^{(j)}=3, \theta=5, h=1, i_{0}^{(1)}=0, i_{1}^{(j)}=0, \gamma_{0}^{(j)}=1$, $\gamma_{1}^{(j)}=0, \psi=5, \epsilon=0.1, \eta=0.1, \delta_{0}^{(j)}=0.1, \delta_{1}^{(j)}=0, \alpha_{0}^{(j, \ell)}=0.8, \alpha_{1}^{(j, \ell)}=0$, $\lambda^{(1,2)}=\lambda^{(2,1)}=1$, and $\tau=500$ for $j=1,2$ and $\ell \neq j$. The initial conditions are $x^{(1)}(0)=0.2, x^{(2)}(0)=0, s^{(1)}(0)=0, s^{(2)}(0)=0, y^{(1)}(0)=0.1$, and $y^{(2)}(0)=0$. In (a), $k=0.4$ and in (b), $k=2.4$. The value of $i_{0}$ is the dependent variable here. in the other two patches, $\delta^{(1)}(t)$ and $\delta^{(3)}(t)$. Migration constrictions are achieved mathematically by setting $\alpha^{(1,3)}=\alpha^{(3,1)}=0$. This is demonstrated graphically in Figure 9. The kinetic proofreading model for T-cells accounts for time-delay in a similar way (George et al., 2005).

One advantage of this model is that it incorporates a delay between the predator leaving $\mathcal{P}^{(1)}$ and arriving at $\mathcal{P}^{(3)}$, and vice versa. Geographically, the patches for the arctic fox, lemming and seal carrion system are often hundreds of kilometers apart (Wrigley and Hatch, 1976) and hence, this delay improves the biological feasibility of the model.

In Figure 10, we look at the effect of the predator mortality rate during migration, $\delta_{0}^{(2)}$, on the dynamics of a three-patch system where the $\mathcal{P}^{(1)}$ is subsidy-free, $\mathcal{P}^{(2)}$ is prey-free and subsidy-free, and $\mathcal{P}^{(3)}$ is prey-free. Increasing $\delta_{0}^{(2)}$ has a stabilizing effect on the dynamics, and ultimately causes predatormortality and prey-survival. This is equivalent to the results in Section 4.3 in which increasing the proportion of predator migratory deaths, $1-\lambda$, stabilised limit cycles and caused predator-extinction.

\subsection{Numerical Simulations}

We now wish to investigate numerically the effect that the amplitude of oscillations of the predator migration rate, $\alpha_{1}$, has on the dynamics of a two-patch system where the first patch is subsidy-free and the second patch is prey-free. The autonomous base cases are displayed in Figure 11(a) where an equilibrium is approached, and Figure 11(b) where a limit cycle is generated.

We examine the effect that $\alpha_{1}$ has on the equilibrium case in Figure 12(a). As $\alpha_{1}$ is increased, limit cycles are generated in the dynamics of the predator, and these limit cycles have quadrennial period. The prey also develop limit cycles with the same period, however their population is very close to zero except for very short periods of time. Note the sharp peaks of the lemming population which typically characterises predators rather than prey (Turchin et al., 2000). For both the predator and prey, the amplitude of the oscillations increases as $\alpha_{1}$ increases.

In Figure 12(b), we examine the effect of $\alpha_{1}$ on the limit cycle in Figure 11(b). As the parameter was varied, there was no significant effect on the maximum and minimum values attained. However, for large values of $\alpha_{1}$, there is an envelope within which the dynamics occur. Consequently, the period of the system changes and becomes quadrennial, with many oscillations occurring within this envelope.

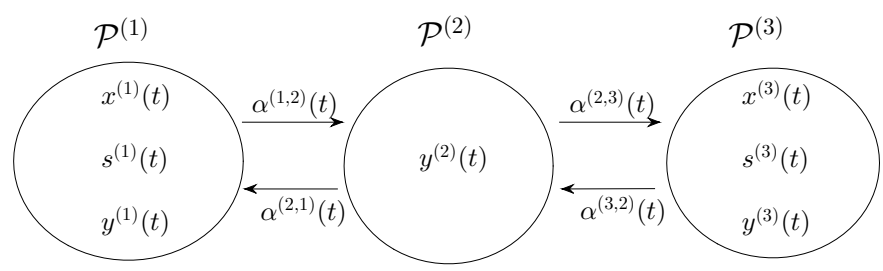

Figure 9: Migration between two patches modelled using a three-patch model. 

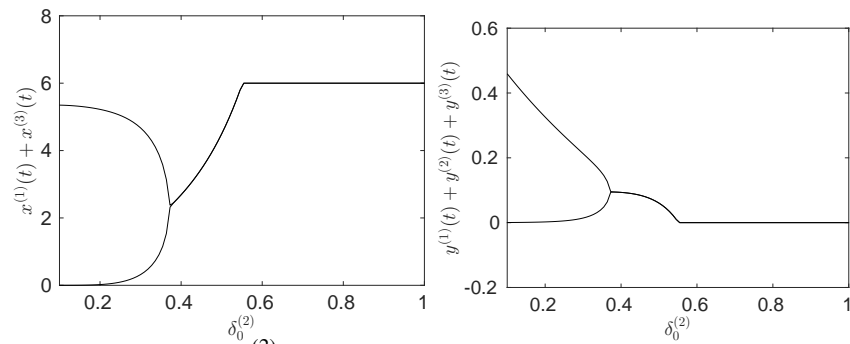

Figure 10: Effect of $\delta_{0}^{(2)}$ on the three-patch autonomous system. We plot the maximum and minimum values of solutions after transient behaviours. The parameters used are $r_{0}^{(j)}=0.1, r_{1}^{(j)}=0, k_{0}^{(j)}=6, \theta=5, h=1, i_{0}^{(1)}=i_{0}^{(2)}=0$, $i_{0}^{(3)}=0.1, i_{1}^{(j)}, \gamma_{0}^{(j)}=1, \gamma_{1}^{(j)}=0, \psi=5, \epsilon=0.1, \eta=0.1, \delta_{0}^{(1)}=\delta_{0}^{(3)}=0.1$, $\delta_{1}^{(j)}=0, \lambda^{(j, \ell)}=1, \alpha_{0}^{(1,2)}=\alpha_{0}^{(2,1)}=\alpha_{0}^{(3,2)}=\alpha_{0}^{(2,3)}=0.8$, and $\alpha_{0}^{(1,3)}=\alpha_{0}^{(3,1)}=0$, for all $j=1,2,3$.

\section{Chaotic Dynamics}

So far, we have discussed equilibrium and limit cycle dynamics of the predator-prey-subsidy system. However, another possible dynamical state of the system could be chaos. Poincaré-Bendixson's theory states that the dynamics of a bounded two-dimensional autonomous system will either approach an equilibrium, or a limit cycle (Drazin, 1992) and therefore chaos does not exist in the autonomous predatorprey system. Possibly the most famous chaotic system is

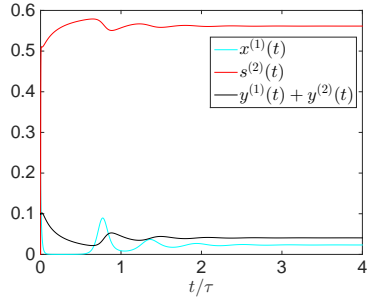

(a) Equilibrium

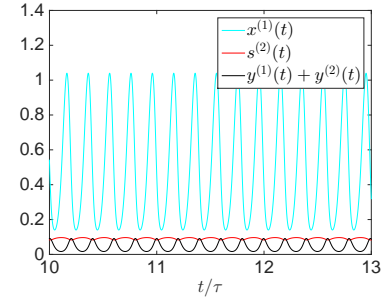

(b) Limit Cycle
Figure 11: The dynamics of the predator-prey-subsidy two-patch system when all the parameters are constant. The parameters are $r_{0}^{(j)}=0.1, r_{1}^{(j)}=0, \theta=5$, $h=1, i_{0}^{(1)}=0, i_{1}^{(j)}=0, \gamma_{0}^{(j)}=1, \gamma_{1}^{(j)}=0, \psi=5, \epsilon=0.1, \eta=0.1, \delta_{0}^{(j)}=0.1$, $\delta_{1}^{(j)}=0, \alpha_{0}^{(j, \ell)}=0.8, \alpha_{1}^{(j, \ell)}$, and $\lambda^{(j, \ell)}=1$ for $j=1,2$ and $\ell \neq j$. The initial conditions are $x^{(1)}(0)=0.2, x^{(2)}(0)=0, s^{(1)}(0)=0, s^{(2)}(0)=0, y^{(1)}(0)=0.1$, and $y^{(2)}(0)=0$. In (a), we use $k_{0}^{(j)}=0.4$ and $i_{0}^{(2)}=0.6$, whilst in (b) we use $k_{0}^{(j)}=2.4$ and $i_{0}^{(2)}=0.1$.

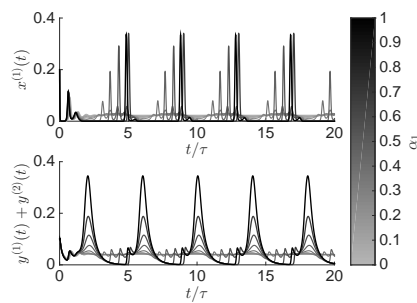

(a)

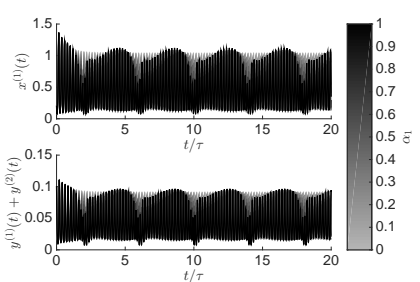

(b)
Figure 12: Examination of the effect that the amplitude of the oscillation of the rate of predator inter-patch migration has on the dynamics of a predatorprey-subsidy system. In (a) we examine how the nonautonomousness of the predator migration rate affects the equilibrium predicted by the autonomous system given in Figure 11(b). In (b), we examine how it affects the limit cycles predicted by the autonomous system given in Figure 11(b). In both examples, we have used $\tau=500$. the Lorenz system, discovered in 1963 (Drazin, 1992; Lorenz, 1963). The Lorenz system is three-dimensional, autonomous, and quadratic, and describes a layer of fluid which is heated from below (Drazin, 1992).

Chaos entered the field of ecology in the late 1970's (Bjønstad, 2015). A biological example of a system which can exhibit chaos is the three-species food chain model where we have a species which acts as a prey to one species, and as a predator to the other (Hastings and Powell, 1991). The threespecies food chain model has a region of parameter space in which the system exhibits chaos.

Nonautonomous predator-prey systems have also been found to exhibit chaos (Rinaldi et al., 1993). Small, periodic perturbations to an autonomous system at equilibrium generated periodic limit cycles instead. In the case of small periodic external forcing, stable limit cycles can be moved into quasi-periodic dynamics on invariant tori. Meanwhile, where limit cycles were previously generated by an autonomous system, chaos was observed when large enough or resonant periodic perturbations to parameters were given. This observation motivates the region of parameter space in which we shall look for chaos in our nonautonomous predator-prey-subsidy system.

\subsection{Test For Chaos}

A common test for chaos is the Maximal Lyapunov Exponent (MLE) Test (Silk et al., 2011; Gottwald and Melbourne, 2003). The Lyapunov exponents of a system describe the rate at which the orbits in phase space of two nearby points converge or diverge as time evolves (Wolf et al., 1985). A system has equally as many Lyapunov exponents as dimensions. If all of the Lyapunov exponents are negative, then the dynamics approaches an equilibrium; if one of the exponents is zero and the rest are negative then we have a limit cycle; and if any Lyapunov exponent is positive then this describes chaotic dynamics (Wolf et al., 1985). The MLE Test classifies the behaviour of a system by considering the sign of the maximal Lyapunov exponent. This is equivalent to determining the existence of a positive exponent. In this paper, we calculate the Lyapunov exponents from a time series data by using code supplied by Steve (1998) which uses techniques described in Wolf et al. (1985); Eckmann and Ruelle (1985). We have modified this code to improve its efficiency. Since we have nonautonomous ODEs, we must convert them to autonomous equations by adding an extra differential equation which describes the evolution of time. This introduces a Lyapunov exponent equal to zero, which we shall ignore in all subsequent work.

\subsection{Chaos In The Primary Model}

We begin our pursuit to discover chaotic behaviours by considering autonomous limit cycles generated by the predatorprey-subsidy system given by Equations (2.1)-(2.3). We allow one of $r_{1}, i_{1}, \delta_{1}$, and $\delta_{2}$ to be non-zero and compute the maximal Lyapunov exponent as we vary this parameter and as we vary $\tau$. Recall that throughout this paper, we have $k_{1}=r_{1}$. 


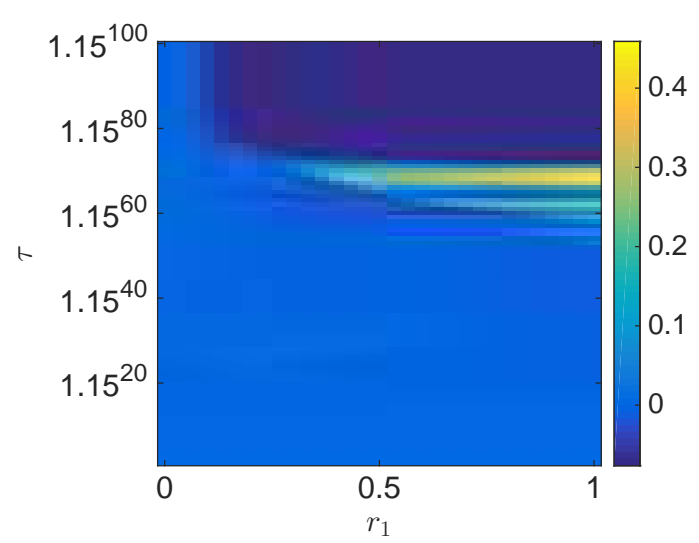

Figure 13: Bifurcation diagrams showing the effect of nonautonomous terms on the autonomous limit cycles in $r_{1}-\tau$ parameter space. The colour at each point gives the value of the MLE for the choice of parameters.

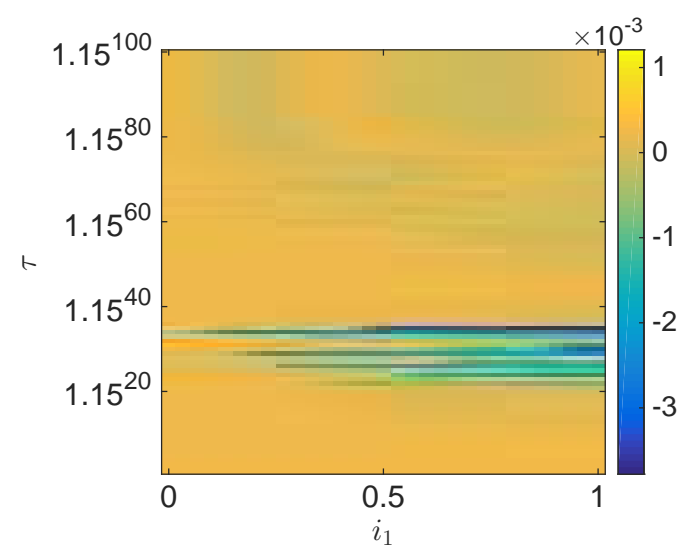

Figure 14: Bifurcation diagrams showing the effect of nonautonomous terms on the autonomous limit cycles in $i_{1}-\tau$ parameter space. The colour at each point gives the value of the MLE for the choice of parameters.

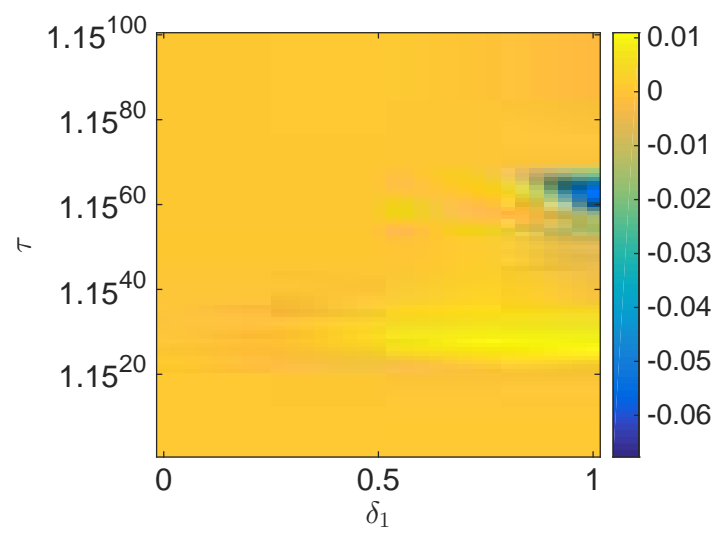

Figure 15: Bifurcation diagrams showing the effect of nonautonomous terms on the autonomous limit cycles in $\delta_{1}-\tau$ parameter space. The colour at each point gives the value of the MLE for the choice of parameters.

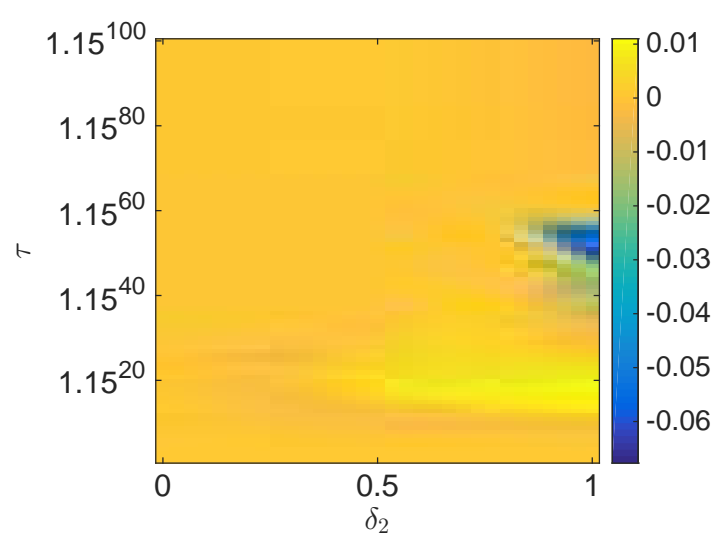

Figure 16: Bifurcation diagrams showing the effect of nonautonomous terms on the autonomous limit cycles in $\delta_{2}-\tau$ parameter space. The colour at each point gives the value of the MLE for the choice of parameters.

For each parameter under consideration, we compute the MLE in parameter space defined by

$$
\left\{p=\frac{a}{30}, \tau=1.15^{b} \mid a=0,1, \ldots, 30, b=1,2, \ldots, 100\right\},
$$

where $p$ represents the parameter from $r_{1}, i_{1}, \delta_{1}$, and $\delta_{2}$ which is under consideration. This grid was chosen so that $p$-space would cover the range $[0,1]$, and so that $\tau$-space would be covered with a logarithmic scale ranging from $\tau \approx 1$ to $\tau \approx 10^{6}$. We run the test for $t=10^{5}$ units of time in order to approximate the MLE. The bifurcation diagrams can be found in Figures 13-16, which display the MLE at each point on this grid.

Our base parameters are $r_{0}=0.1, r_{1}=0, k_{0}=2, \theta=5$, $h=1, i_{0}=0.05, i_{1}=0, \gamma_{0}=1, \gamma_{1}=0, \psi=5, \epsilon=0.1$, $\eta=0.1, \delta_{0}=0.1, \delta_{1}=0$, and $\delta_{2}=0$. The initial conditions are $x(0)=0.1, s(0)=0, y(0)=0.1$. For our autonomous base case, the MLE is 0 , confirming that limit cycles are generated (Wolf et al., 1985).

Choosing regions where chaos is suggested by our bifurcation diagrams, we investigate the effect of the nonautonomous contributions to autonomous limit cycles. In each case, we construct the phase portrait of the dynamics for large enough time for transient behaviours to become insignificant. We also provide a graph displaying how the dynamics evolve in time. Finally, we provide a graph which shows the evolution of the estimations of the Lyapunov exponents, in order to demonstrate that one of the exponents is positive. Only the larger two Lyapunov exponents are displayed on each graph, because the third exponent was sufficiently negative to be unimportant in the investigation of chaos. Note that if the reader repeated these simulations with a different computer or numerical scheme, the chaotic nature of the system would mean that the reader would be unlikely to obtain exactly the same results as the author. However, the same general phenomena should be observed.

In Figure 17, we investigate the effect of time dependent prey growth rate, $r(t)$, and keep $\frac{r(t)}{k(t)}$ a constant. We have used the parameters $r_{1}=\frac{14}{15}$, and $\tau=1.15^{28} \approx 50$. We observe chaotic behaviour restricted to a two-dimensional manifold. The MLE 
is 0.0106 and this means that we have strong evidence that we have indeed found chaotic behaviour.

We investigate the dynamics obtained with a time-dependent subsidy input rate, $i(t)$, in Figure 18 . The parameters chosen are $i_{1}=\frac{14}{15}$, and $\tau=1.15^{28} \approx 50$. These parameters lie in the most yellow region of the bifurcation diagram for $i_{1}-\tau$, nested amongst the blue region where equilibrium is attained. The dynamics obtained are restricted to the surface of a torus with annulus cross-section in the $x-y$ plane. The MLE is 0.0012 . This is perhaps weak evidence for chaos in some of the parameter regimes adjacent to these, while for the parameter regimes used here, we find that this is one such case where the small periodic external forcing due to $i_{1}$ pushes stable limit cycles into quasi-periodic dynamics on invariant tori.

Finally, we investigate whether the predator mortality rate, $\delta(t)$, can cause chaotic behaviours in the system in Figures 19-20. Figure 19 uses parameters $\delta_{1}=0.8, \delta_{2}=0$, and $\tau=1.15^{28} \approx 50$, and Figure 20 uses parameters $\delta_{1}=0$, $\delta_{2}=0.8, \tau=1.15^{20} \approx 16.4$. The MLE is 0.094 and 0.074 for these figures, respectively. We conclude with strong evidence that these dynamics are indeed chaotic.

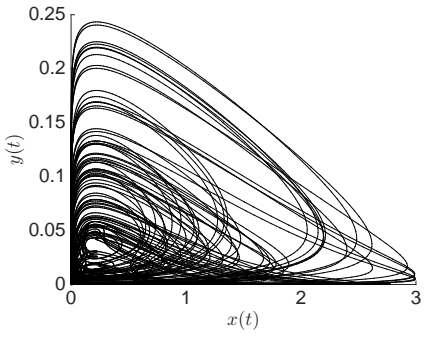

(a)

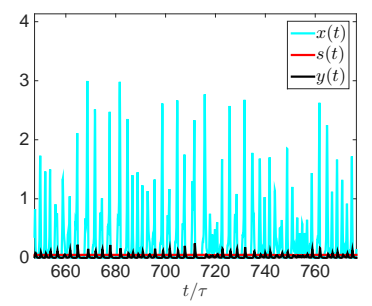

(c)

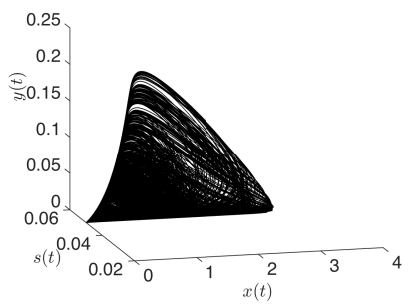

(b)

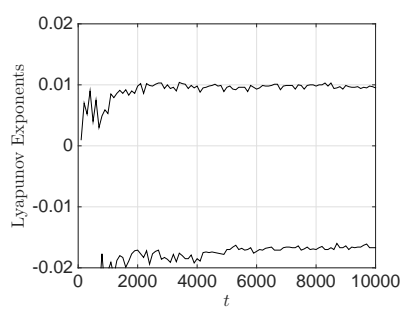

(d)
Figure 17: Investigation of chaos for time-dependent prey growth rate, $r(t)$, and carrying capacity, $k(t)$. In (a), we show predator-prey phase space for $t \in$ $\left[9.9 \times 10^{4}, 10^{5}\right]$. In (b) we show the $3 D$ space diagram for $t \in\left[10^{4}, 10^{5}\right]$. We show the time series of the dynamics in (c). Finally, in (d), we show a graph of the Lyapunov exponents being calculated. Only the largest two exponents are displayed because the third one is sufficiently negative to give no indication about chaos. The parameters used are $r_{0}=0.1, r_{1}=\frac{14}{15}, k_{0}=2, \theta=5, h=1$, $i_{0}=0.05, i_{1}=0, \gamma_{0}=1, \gamma_{1}=0, \phi=5, \epsilon=0.1, \eta=0.1, \delta_{0}=0.1, \delta_{1}=0$, $\delta_{2}=0$, and $\tau=1.15^{28} \approx 50$.

\subsection{Chaos In The n-Patch Model}

We now look for interesting behaviours exhibited in the twopatch model given by Equations (2.4)-(2.6), when we consider the prey migration rate, $\alpha^{(j, \ell)}(t)$, to be time-dependent. Our two patch model has spatially separated prey and subsidy and exhibits limit cycles. Our autonomous base case uses the parameters $r_{0}^{(j)}=0.1, r_{1}^{(j)}=0, k_{0}^{(j)}=2.4, \theta=5, h=1, i_{0}^{(1)}=0$,

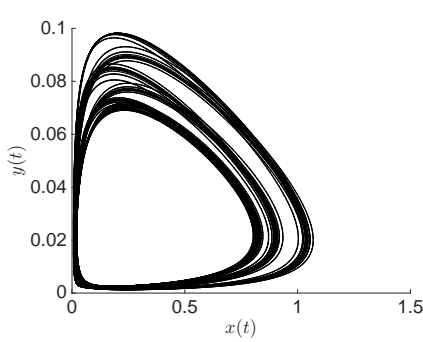

(a)

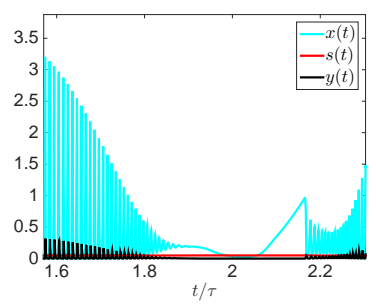

(c)

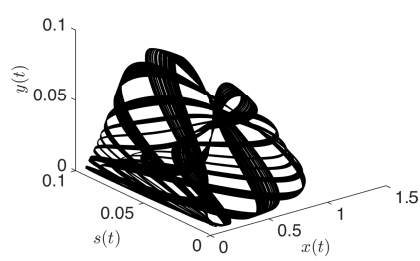

(b)

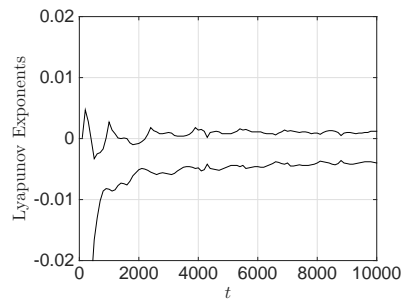

(d)
Figure 18: Investigation of chaos for time-dependent subsidy input rate, $i(t)$. Panels (a)-(d) are the same as in Figure 17. The parameters used are $r_{0}=0.1$, $r_{1}=0, k_{0}=2, \theta=5, h=1, i_{0}=0.05, i_{1}=\frac{14}{15}, \gamma_{0}=1, \gamma_{1}=0, \phi=5, \epsilon=0.1$, $\eta=0.1, \delta_{0}=0.1, \delta_{1}=0, \delta_{2}=0$, and $\tau=1.15^{28} \approx 50$.

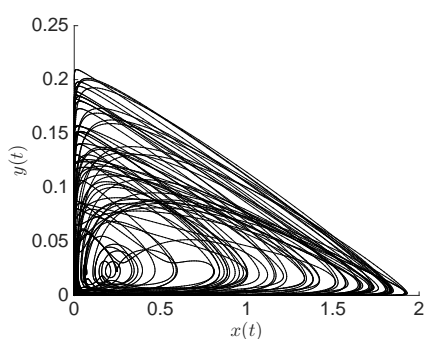

(a)

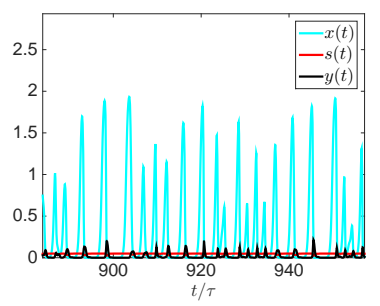

(c)

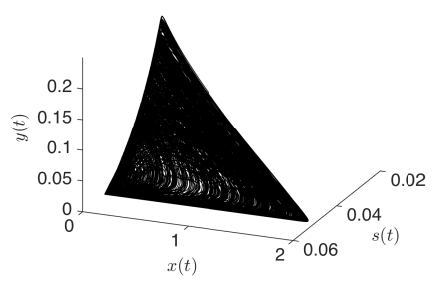

(b)

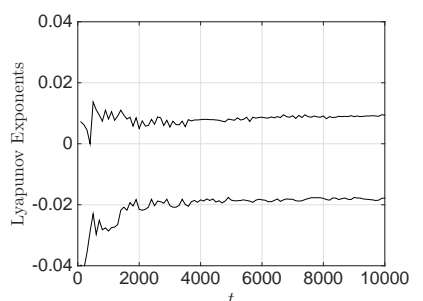

(d)
Figure 19: Investigation of chaos for time-dependent predator mortality rate, $\delta(t)$. Panels (a)-(d) are the same as in Figure 17. The parameters used are $r_{0}=0.1, r_{1}=0, k_{0}=2, \theta=5, h=1, i_{0}=0.05, i_{1}=0, \gamma_{0}=1, \gamma_{1}=0, \phi=5$, $\epsilon=0.1, \eta=0.1, \delta_{0}=0.1, \delta_{1}=0.8, \delta_{2}=0$, and $\tau=1.15^{28} \approx 50$.

$i_{0}^{(2)}=0.1, i_{1}^{(j)}=0, \gamma_{0}^{(j)}=1, \gamma_{1}^{(j)}=0, \psi=5, \epsilon=0.1, \eta=0.1$, $\delta_{0}^{(j)}=0.1, \delta_{1}^{(j)}=0, \alpha_{0}^{(j, \ell)}=0.8, \alpha_{1}^{(j, \ell)}=0, \lambda^{(j, \ell)}=1$ for $j=1,2$ and $\ell \neq j$. The initial conditions are $x^{(1)}(0)=0.1, x^{(2)}(0)=0$, $s^{(1)}(0)=s^{(2)}(0)=0, y^{(1)}(0)=y^{(2)}(0)=0.1$. It has MLE equal to 0 . Therefore, the base case does not exhibit chaos.

We look for chaos in the nonautonomous system by creating a bifurcation diagram for $\alpha_{1}-\tau$ space. This is given in Figure 21. We pick the parameters $\alpha_{1}=1$, and $\tau=1.15^{39} \approx 233$ as these are in the region where the MLE is largest. Figure 22 shows the dynamics in the phase space from various angles, 


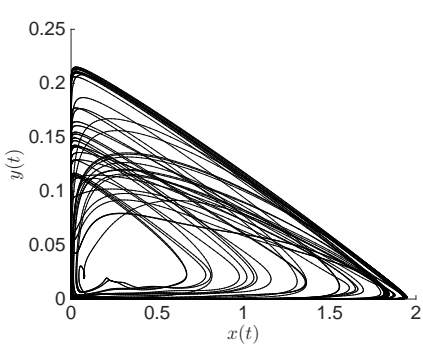

(a)

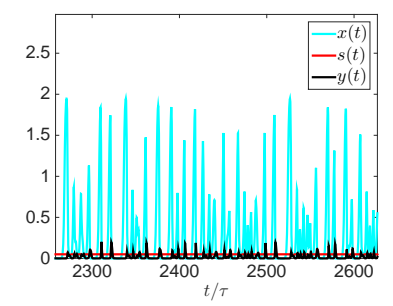

(c)

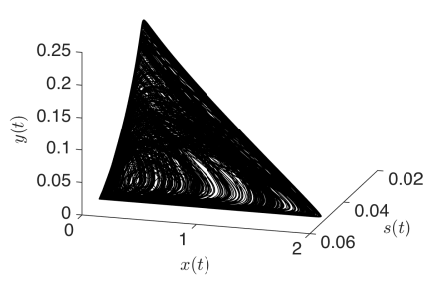

(b)

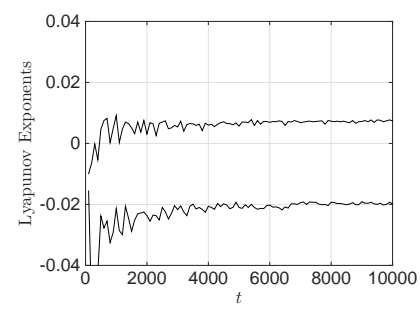

(d)

Figure 20: Investigation of chaos for time-dependent predator mortality rate, $\delta(t)$. Panels (a)-(d) are the same as in Figure 17. The parameters used are $r_{0}=0.1, r_{1}=0, k_{0}=2, \theta=5, h=1, i_{0}=0.05, i_{1}=0, \gamma_{0}=1, \gamma_{1}=0, \phi=5$, $\epsilon=0.1, \eta=0.1, \delta_{0}=0.1, \delta_{1}=0, \delta_{2}=0.8$, and $\tau=1.15^{20} \approx 16.4$.

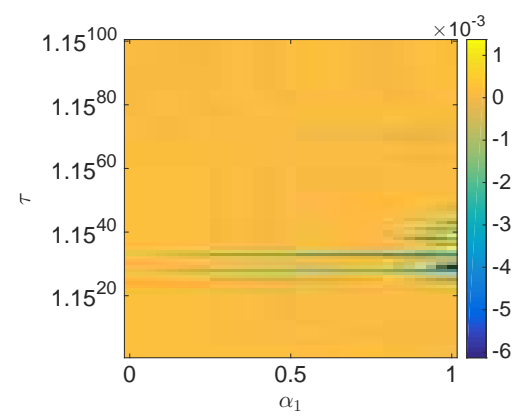

Figure 21: Bifurcation diagram showing the effect of nonautonomous predator migration rate on autonomous cycles in the two-patch model with spatially separated prey and subsidy. The colour at each point gives the MLE for that particular choice of parameters.

how the dynamics evolve in time, and a graph showing the evolution of our approximation of the MLE. Only the largest two Lyapunov exponents are displayed because the other two are sufficiently negative as to not influence the classification of the system. The MLE found is 0.0014 , suggesting these dynamic are chaotic.

\section{Biological Discussion}

\subsection{Biological Implications}

The analysis carried out in this paper is motivated by the arctic fox, lemming, seal carrion ecological system in the Arctic. Similar analysis and results are applicable to other systems, such as the wolf, deer, salmon carcass system (Darimont et al., 2008; Adams et al., 2010); systems where river otters (Lontra canadensis) have an allochthonous subsidy (Ben-David et al., 2005); and systems where puma (Puma concolor), a natural predator, benefits from mule deer (Odocoileus hemionus) carcass (Bauer et al., 2005). The Primary and $n$-Patch Models

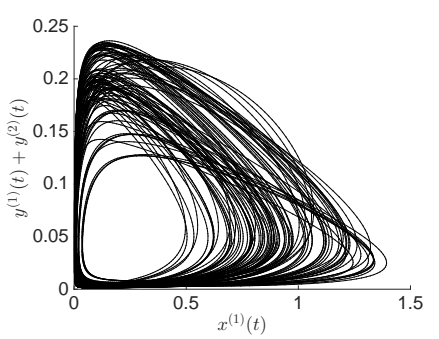

(a)

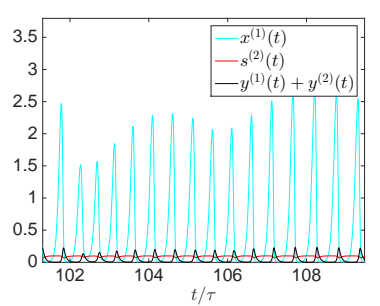

Figure 22: Investigation of chaos for time-dependent predator migration rate, $\alpha^{(j, \ell)}(t)$. In (a), we give a phase portrait for $t \in\left[9.9 \times 10^{4}, 10^{5}\right]$. In (b) we show the phase space diagram for $t \in\left[10^{4}, 10^{5}\right]$. We show the time evolution of the dynamics in (c). Finally, in (d), we show a graph of the Lyapunov exponents being calculated. Only the largest two exponents are displayed because the third one is sufficiently negative to give no indication about chaos. The parameters used are $r_{0}^{(j)}=0.1, r_{1}^{(j)}=0, k_{0}^{(j)}=2.4, \theta=5, h=1, i_{0}^{(1)}=0, i_{0}^{(2)}=0.1, i_{1}^{(j)}=0$, $\gamma_{0}^{(j)}=1, \gamma_{1}^{(j)}=0, \phi=5, \epsilon=0.1, \eta=0.1, \delta_{0}^{(j)}=0.1, \delta_{1}^{(j)}=1, \alpha_{0}^{(j, \ell)}=0.8$, $\alpha_{1}^{(j, \ell)}=1, \lambda^{(j, \ell)}=1$, and $\tau=1.15^{39} \approx 233$, for $j=1,2$ and $\ell \neq j$.

could be good models of these systems, with sensible choices for the parameters describing the prey growth rate and carrying capacity, the subsidy input rate and decay rate, and the predator mortality and migration rates.

In our particular motivating example, biologists have noted strange oscillations in the population size of lemmings (Roth, 2002, 2003; Turchin et al., 2000; Fuller et al., 1975; Lindström et al., 2001) which have a period of approximately $3-4$ years. It was suggested in Lindström et al. (2001) that this could be due to climactic effects, which in turn suggests a temporal study of the system is necessary in order to understand this phenomenon. In Section 3, we observe that time-varying parameters generate limit cycles in the dynamics of the system. Moreover, the dynamics in some of the examples considered are sensitive to the choice of parameters, as in Figure 3(b). In nature, we do not expect our choices of parameters to be precise, but hope they are a good approximation of natural phenomena. Therefore, the lengths of the periods of the cycles is likely to be influenced by the irregularity of nature and thus generate aperiodic cycles in the size of the lemming populations.

The importance of this paper to a mathematical biologist is the inclusion of non-autonomous terms in a population dynamics model. We found that implicitly including temporal effects in our model resulted in limit cycles instead of autonomously predicted equilibria when the amplitude of the oscillations was small. When the amplitude of oscillation was sufficiently large, we observed cases when previously predicted positive equilibria resulted in extinction of one, or both, of the predator and prey. Often, this was accompanied by boom and bust type dy- 
namics which have been observed in nature (Barnes, 2002; Stott et al., 2010; Lee, 1974; Kingsford et al., 1999; Dickman et al., 2010). When the autonomous model predicted limit cycles, we found that our non-autonomous models could predict more interesting dynamics including aperiodic behaviours and even chaos. Therefore, the autonomous and non-autonomous models sometimes fundamentally disagreed in the dynamics of the system. Similar results were observed for a two-dimensional predator-prey model in Rinaldi et al. (1993).

In Sections 3-4 we examined the implications of our Primary and $n$-Patch Models, respectively. Our first result in both sections was that the dynamics of the populations are bounded and non-negative. Biologically, we cannot attain a negative population, and we also observe that limited resources and competition result in populations which do not blow up in size to infinity. This implies that our model is reasonable to describe the predator-prey-subsidy system. In our proofs, we had to assume that prey growth rate, carrying capacity, and subsidy input rate were bounded above, and that the subsidy decay rate and the predator mortality rate were bounded below away from zero. These are all biologically reasonable assumptions, for example the carrying capacity is bounded above because even during a summer with a particularly high abundance of plant-life for the prey to consume, the environment can only support a finite number of prey.

We examined the effect of introducing a subsidy into the predator-prey model for both the autonomous Primary, and autonomous $n$-Patch Model. For both the Primary and $n$-Patch Models, we considered two cases - one where the subsidy-free system approaches a stable positive equilibrium, and the other where it approaches a stable limit cycle. For the stable equilibria, we found that introducing subsidy into the system benefited the predator, but consequently harmed the prey as there was greater predation pressure. Therefore the nutritional gain to the system was restrained by the damage caused to the prey. For the limit cycles, increasing the rate at which the subsidy was inputted into the system could both increase and decrease the peaks and troughs of the limit cycles. For large enough subsidy input rate, the limit cycles were replaced by equilibria. In all four cases examined, there was greater benefit to the predator population of increasing the subsidy input rate once the prey had become extinct because now the predator could profit fully from the nutritional gain of the increased subsidy input rate.

We considered the effect of the predator mortality rate due to migration on the population dynamics in three distinct manners: in the Primary Model, this was described by the parameter $\delta_{2}$ in the time-dependent parameter $\delta(t)$; in the $n$-Patch Model, the parameter $1-\lambda$ denoted the proportion of the migrating predators which perish en-route; in the three-patch model, the increased predator-mortality parameter in the patch which did not contain prey or subsidy accounted for increased migratory mortality rates. We found that increasing the proportion of migratory deaths could ultimately drive the predator to extinction, and thus benefit the prey. We also found that migration mortality could induce limit cycles.

The models, analysis, and results in this paper are important for ecological conservation. In our motivating example, we wish to ensure that the arctic fox population and the lemming population are both able to persist. One example of a way in which human intervention interferes in this ecosystem is in the trapping of the arctic fox during migration for the fur industry. Whilst naïvely, we may suspect that banning the fur industry would have a positive effect on the ecosystem, we may find out that without the fur industry we would experience boom and bust behaviours in which an increase of the arctic fox population drives the lemmings to extinction, which in turn drives the arctic fox to extinction, as seen in Figure 5(c). Conversely, over-trapping may also drive the arctic fox to extinction, as in Figure 10. Therefore, the fur industry should use these models to produce regulations for trapping in order to protect the wildlife. Statistical studies of the effects of the fox hunting ban in the United Kingdom can be used for reference (Baker et al., 2002; Baker and Harris, 2006; Aebischer et al., 2003). This merits further work.

Our analysis has highlighted the importance of the polar bear as a provider of the subsidy for the arctic fox. Therefore, preservation of the arctic foxes and lemmings is directly related to the preservation of the polar bear. Polar bear dynamics has been found to correlate with arctic fox dynamics (Roth, 2003). Irregular behaviour of the polar bear could result in an increased subsidy rate which could harm the lemming population by benefiting the arctic foxes, or a decreased subsidy rate which could harm the arctic fox population and consequently benefit the lemmings. Accordingly, wildlife activists interested in our particular ecosystem should also be interested in the polar bears' ecosystem. Should the polar bear behave irregularly, conservationalists should provide or remove some subsidy from the system to ensure the continuation of the ecosystem.

In the final section of our research, we found that seasonality could cause chaos in our system. In fact, chaos has been observed in nature in populations of arctic lemmings, but not in populations of subarctic voles, which strongly suggests that seasonality is the cause of this chaos (Oksanen and Oksanen, 1992). Turchin discovered similar behaviours by using Lyapunov exponents to show that chaotic behaviour of microtine populations was possible at latitudes further north than $60^{\circ} \mathrm{N}$ whilst limit cycles were observed at more temperate latitudes (Falck et al., 1995; Turchin, 1993). There are some papers which suggest different mechanisms are responsible for the dynamics such as Christian (1978); Krebs et al. (1973); Seldal et al. (1994).

\subsection{Further Work}

In this section, we suggest potential directions in which this paper could be extended in further work. The directions are: extending the models to include the effects of global warming; accounting for the fact that an intelligent predator does not migrate away from a patch with abundant food supply; explicitly considering the time required to migrate between patches; modelling the dynamics within each patch with PDEs; and including the dynamics between the polar bears and live seals explicitly in the model. 


\subsubsection{Accounting for Global Warming}

Long term dynamics of the predator-prey-subsidy system may be influenced by changes in global temperature. For example, hot temperatures melt the arctic ice caps and therefore affect the rate at which the seal carrion is produced and the rate of predator inter-patch migration; and increases the rate at which the subsidy decays.

As such, one could investigate the effect of having non-zero parameter $m$ in the parameter given in Equation (2.13) or Equation (2.14). In line with the average annual rise in global temperature, a possible value of this parameter could be (Root et al., 2003)

$$
m=0.006 K \text { year }^{-1} \text {. }
$$

where $K$ denotes Kelvins.

Results given in such studies could show that populations which were thought to have stable, positive dynamics are actually approaching extinction.

\subsubsection{Intelligent Predators}

In our $n$-Patch Model, the predator migrates between patches with the highest rate of migration occurring every four years. Migration occurs independently of whether each patch has sufficient food to sustain the predator population. In our model, the predator may migrate away from a fruitful patch which is not realistic behaviour. By introducing nonlinear diffusion, we can account for the intelligence of the predator.

\subsubsection{Delayed Migration}

Migration thus far has been assumed to occur instantaneously with the exception of the three-patch model in Section 4.5. The arctic foxes have been found to cover as much as $1000 \mathrm{~km}$ whilst migrating (Wrigley and Hatch, 1976). Therefore, a delay equation may be better suited to describe migration.

\subsubsection{Intra-Patch Migration}

In addition to inter-patch migration, we could also consider intra-patch movements. This would evolve our equations into PDEs and would describe the behaviour within each patch in a more accurate way.

\subsubsection{Predator-Quarry-Scavenger-Prey-Subsidy Model}

We have discussed the important role of the polar bear on the ecological system of the arctic fox, lemming, and seal carrion as the primary predator of seals and therefore the provider of the carrion. However, we do not investigate the dynamics of the predator-prey system of the polar bear and seal explicitly. Instead, we approximate the effects implicitly in the time dependent parameter, $i(t)$, representing the input rate of the subsidy.

Instead, we could consider a system of five coupled ODEs which describe the dynamics of the polar bear (predator), seal (quarry), arctic fox (scavenger), lemming (prey), and seal carrion (subsidy), where the predator and quarry equations can be decoupled and obey a predator-prey equation, and the scavenger-prey-subsidy equations are as in this paper, with the input rate of subsidy replaced with a term proportional to the rate of consumption of the quarry by the predator.

\section{Conclusions}

We have modeled predator-prey-subsidy systems, motivated by the system in the Arctic which comprises of arctic foxes; lemmings; and seal carcasses provided by polar bears. In particular, we have focused on the importance of seasonality to this system due to the the significant differences in the ecosystem during winter and summer months. These include increased subsidy input rate in the winter due to increased seal predation by polar bears, and increased subsidy decay rate in the summer due to the higher temperatures. Our analysis is not limited to this particular ecosystem and is applicable to any other system which obeys predator-prey-subsidy behaviours.

Due to the importance of the resource subsidy in our system, demonstrated in Figures 1 and 8, we cannot apply the Lotka-Volterra Equations, or any variations of this predatorprey model. Instead, we developed two new models which were inspired by the models presented by Nevai and Van Gorder (2012). The key modifications to these models were the explicit consideration of seasonality, and the implicit consideration of the risks of predator migration. The factors which we decided to model by time-dependent parameters were the prey growth rate and carrying capacity, the subsidy input rate and decay rate, and the predator mortality rate and rate of migration. In particular, the subsidy decay rate is a function of temperature, which in turn is a function of time. The form of these time-dependencies was motivated by the biology of the system. We developed 3dimensional, and $3 n$-dimensional, systems of nonlinear, nonautonomous ODEs to analyse. These models were named the Primary Model, and the $n$-Patch Model, respectively.

The key difference between the Primary and the $n$-Patch Model is the way that they account for the spatial layout of the Arctic. In the Primary Model, we consider the ecosystem as a whole and assume that the predator is able to migrate between patches instantaneously in order to access any of its food sources. As such, we assume that the Arctic is a homogeneous environment. We accounted for the increase in predator deaths during migration by choosing a function which describes the predator mortality rate to have quadrennial oscillations to match the timescale in which migration occurs. Contrastingly, in the $n$-Patch Model, each spatially separated patch in the Arctic is modeled by an explicitly spatially-separated patch with a heterogeneous environment. The quadrennial migration is explicitly included in the time-dependent migration rate, and although migration is still considered to be instantaneous, there is now a mortality cost explicitly associated with migration. When the $n$-Patch Model consisted of homogeneous patches with identical initial conditions, the system was equivalent on both a micro and macro level to the Primary Model. Therefore, we only considered patches with heterogeneous conditions or distinct initial conditions when investigating the $n$-Patch Model.

We sought to investigate the effect of introducing prey into a prey-free patch of a two-patch model with spatially separated prey and subsidy. We found conditions under which, the newly introduced prey would die out, and conditions under which it would thrive. In the example considered, we found that the survival of the new prey came at a cost to the population size 
of the prey in the patch which is subsidy-free, but increased the total prey population size of the system.

In Figures 3, 5, and 12, we found that including seasonality in our model could fundamentally alter the classification of solutions attained when only considering parameter averages. For example, when an autonomous system predicts an equilibrium, we found that limit cycles could be induced (e.g. Figure 3(a)), and even seasonality-induced extinction was possible (e.g. Figure 3(c)). The temperature in the Arctic has a range of $32^{\circ} \mathrm{C}$ (Danish Meteorological Institute, 2015) and therefore, it is very important to include seasonality in this particular model of species interaction.

We found the solution to the equations via an asymptotic series, which could be expressed analytically up to first order provided that the corresponding autonomous model approached an equilibrium, and the magnitude of the parameter oscillations was small. Limit cycles could be constructed in this manner. Moreover, limit cycles could be generated by migrationinduced predator mortality rates (see Figure 6).

We further found regions in parameter space where deterministic chaotic behaviour is possible, as verified by the MLE Test. These regions are displayed in Figures ?? - 21. The chaos found was a result of interactions between the natural oscillations of a system with constant parameters, and the oscillations of our time-dependent parameters. This is another example of the way in which seasonality can fundamentally change the nature of our solutions.

\section{Acknowledgments}

DL gratefully acknowledges the support of StarLeaf Ltd. HAH gratefully acknowledges the support of EPSRC Fellowship EP/K041096/1.

\section{References}

Abrams, P. A., 2000. The evolution of predator-prey interactions: Theory and evidence. Annual Review of Ecology and Systematics 31, 79-105.

Adams, L. G., Farley, S. D., Stricker, C. A., Demma, D. J., Roffler, G. H., Miller, D. C., Rye, R. O., 2010. Are inland wolf-ungulate systems influenced by marine subsidies of pacific salmon? Ecological Applications 1 (20), 251-262.

Aebischer, N. J., Baker, S. E., Johnson, P. J., Macdonald, D. W., Reynolds, J. C., 2003. Ecology: Hunting and fox numbers in the United Kingdom. Nature 400 (423), 400-400.

Audet, A. M., Robins, C., Larivère, S., 2002. Alopex lagopus. American Society of Mammalogists 713, 1-10.

Ayala, F. J., Gilpin, M. E., Ehrenfeld, J. G., 1973. Competition between species: Theoretical models and experimental tests. Theoretical Population Biology 4 (3), 331-356.

Baker, P. J., Harris, S., 2006. Does culling reduce fox (Vulpes vulpes) density in commercial forests in Wales, UK? European Journal of Wildlife Research 52 (2), 99-108.

Baker, P. J., Harris, S., Webbon, C. C., 2002. Ecology: Effect of British hunting ban on fox numbers. Nature 419 (878), 34-34.

Barnes, R. F. W., 2002. The bushmeat boom and bust in West and Central Africa. Oryx 36 (3), 236-242.

Bauer, J. W., Logan, K. A., Sweanor, L. L., Boyce, W. M., 2005. Scavenging behavior in puma. The Southwestern Naturalist 50 (4), 461-471.

Ben-David, M., Blundell, G. M., Kern, J. W., Maier, J. A. K., Brown, E. D., Jewett, S. C., 2005. Communication in river otters: Creation of variable resource sheds for terrestrial communities. Ecology 86 (5), 1331 - 1345.
Berryman, A. A., 1992. The origins and evolution of predator-prey theory. Ecology 73 (5), 1530-1535.

Bjønstad, O. N., 2015. Nonlinearity and chaos in ecological dynamics revisited. Proceedings of the National Academy of Sciences 112 (20), 6252-6253.

Borgerson, S. G., 2008. Arctic meltdown: The economic and security implications of global warming. Foreign Affairs 87 (2), 63-77.

Christian, J. J., 1978. Neurobehavioral endocrine regulation of small mammal populations. Populations of small mammals under natural conditions 10 (3), $143-158$.

Danish Meteorological Institute, 2015. Arctic temperatures. http://ocean. dmi.dk/arctic/meant80n.uk.php, accessed: 2015-05-19.

Darimont, C. T., Paquet, P. C., Reimchen, T. E., 2008. Spawning salmon disrupt trophic coupling between wolves and ungulate prey in coastal British Columbia. BMC Ecology 8 (14), 1-12.

de Valdez, M. R. W., Nimmo, D., Betza, J., Gong, H.-F., James, A. A., Alphey, L., IV, W. C. B., 2011. Genetic elimination of dengue vector mosquitoes. Proceedings of the National Academy of Sciences 108 (12), 4772-4775.

Dickman, C. R., Greenville, A. C., Beh, C.-L., Tamayo, B., Wardle, G. M., 2010. Social organization and movements of desert rodents during population booms and busts in Central Australia. Journal of Mammalogy 91 (4), 798-810.

Drazin, P. G., 1992. Nonlinear Systems. Cambridge University Press, Cambridge, UK.

Eckmann, J. P., Ruelle, D., 1985. Ergodic theory of chaos and strange attractors. Reviews of Modern Physics 57 (3), 617-656.

Falck, W., Bjørnstad, O. N., Stenseth, N. C., 1995. Voles and lemmings: chaos and uncertainty in fluctuating populations. Proceedings of the Royal Society of London B: Biological Sciences 262 (1365), 363-370.

Fuller, W. A., Martell, A. M., Smith, R. F. C., Speller, S. W., 1975. High-arctic lemmings, Dicrostonyx groenlandicus II. Demography. Canadian Journal of Zoology 53 (6), 867-878.

George, A. J. T., Stark, J., Chan, C., 2005. Understanding specificity and sensitivity of T-cell recognition. Trends in Immunology 26 (12), 653-659.

Gottwald, G. A., Melbourne, I., 2003. A new test for chaos in deterministic systems. Proceedings of the Royal Society of London A: Mathematical, Physical and Engineering Sciences 460 (2042), 603-611.

Hastings, A., Powell, T., 1991. Chaos in a three-species food chain. Ecology 72 (3), 896-903.

Holling, C. S., 1959. The components of predation as revealed by a study of small mammal predation of the european pine sawfly. Canadian Entomologist 91 (5), 293-320.

Holling, C. S., 1966. The functional response of invertebrate predators to prey density. Memoirs of the Entomological Society of Canada 98 (S48), 5-86.

Holmes, E. E., Lewis, M. A., Banks, J. E., Veit, R. R., 1994. Partial differential equations in ecology: Spatial interactions and population dynamics. Ecology 75 (1), 17-29.

Hutson, V., Vickers, G. T., 1982. A criterion for permanent coexistence of species, with an application to a two-prey one-predator system. Mathematical Biosciences 63 (2), 253-269.

Kareiva, P., Mullen, A., Southwood, R., 1990. Population dynamics in spatially complex environments: Theory and data [and discussion]. Philosophical Transactions of the Royal Society B: Biological Sciences 330 (1257), 175-190.

Kingsford, R. T., Curtin, A. L., Porter, J., 1999. Water flows on Cooper Creek in arid Australia determine boom and bust periods for waterbirds. Biological Conservation 88 (2), 231-248.

Klebanoff, A., Hastings, A., 1994. Chaos in one-predator, two-prey models: General results from bifurcation theory. Mathematical Biosciences 122 (2), 221-233.

Krebs, C. J., Gaines, M. S., Keller, B. L., Meyer, J. H., Tamarin, R. H., 1973. Population cycles in small rodents. Science 179 (4068), 35-41.

Lee, R., 1974. The formal dynamics of controlled populations and the echo, the boom and the bust. Demography 11 (4), 563-585.

Levin, S. A., 1976. Population dynamic models in heterogeneous environments. Annual Review of Ecology and Systematics 7, 287-310.

Lima, S. L., 2002. Putting predators back into behavioral predator-prey interactions. Trends in Ecology \& Evolution 17 (2), 70-75.

Lindström, J., Ranta, E., Kokko, H., Lundberg, P., Kaitala, V., 2001. From arctic lemmings to adaptive dynamics: Charles Elton's legacy in population ecology. Biological Reviews of the Cambridge Philosophical Society 76 (1), 129-158. 
Lorenz, E. M., 1963. Deterministic nonperiodic flow. Journal of the Atmospheric Sciences 20 (2), 130-141.

Lotka, A. J., 1925. Elements of Physical Biology. Williams \& Wilkins, Baltimore, Maryland, USA.

Malthus, T. R., Appleman, P., 1976. An Essay on the Principle of Population. Norton, New York.

MATLAB, 2014. Version 8.4 (R2014b). The MathWorks Inc., Natick, Massachusetts.

Meyer, J., Anderson, B., D.O.Carter, 2013. Seasonal variation of carcass decomposition and gravesoil chemistry in a cold (dfa) climate. Journal of Forensic Science 58 (5), 1175-1182.

Nevai, A. L., Van Gorder, R. A., 2012. Effect of resource subsidies on predatorprey population dynamics: a mathematical model. Journal of biological dynamics $6(2), 891-922$.

Oksanen, L., Oksanen, T., 1992. Long-term microtine dynamics in north Fennoscandian tundra: the vole cycle and the lemming chaos. Ecography 15 (2), 226-236

Plumptre, A. J., Fuller, R. A., A. Rwetsiba and, F. W., Kujirakwinja, D., Driciru, M., Nangendo, G., Watson, J. E. M., Possingham, H. P., 2014. Efficiently targeting resources to deter illegal activities in protected areas. Jounal of Applied Ecology 51 (3), 714-725.

Prestrud, P., 1991. Adaptions by the arctic fox (Alopex logopus) to the polar winter. Arctic 44 (2), 132-138.

Rinaldi, S., Muratori, S., Kuznetsov, Y., 1993. Multiple attractors, catastrophes and chaos in seasonally perturbed predator-prey communities. Bulletin of Mathematical Biology 55 (1), 15-35.

Root, T. L., Price, J. T., Hall, K. R., Schneider, S. H., Rosenzweig, C., Pounds, J. A., 2003. Fingerprints of global warming on wild animals and plants. Nature 421 (6918), 57-60.

Roth, J. D., 2002. Temporal variability in arctic fox diet as reflected in stablecarbon isotopes; the importance of sea ice. Oecologia 133 (1), 70-77.

Roth, J. D., 2003. Variability in marine resources affects arctic fox population dynamics. Journal Of Animal Ecology 72 (4), 668-676.

Seldal, T., Andersen, K.-J., Högstedt, G., 1994. Grazing-induced proteinase inhibitors: a possible cause for lemming population cycles. Oikos 70 (1), 3-11.

Sih, A., 1984. Prey refuges and predator-prey stability. Theoretical Population Biology 31 (1), 1-12.

Silk, D., Kirk, P. D. W., Barnes, C. P., Toni, T., Rose, A., Moon, S., Dallman, M. J., Stumpf, M. P. H., 2011. Designing attractive models via automated identification of chaotic and oscillatory dynamical regimes. Nature Communications 2 (489), 1-6.

Solomon, M. E., 1949. The natural control of animal population. Journal of Animal Ecology 18 (1), 1 - 35.

Steve, W. K., 1998. Lyapunov exponents toolbox. http://uk.mathworks. com/matlabcentral/fileexchange/233-let, mATLAB Central File Exchange, Retrieved 30-06-2015.

Stott, I., Franco, M., Carslake, D., Townley, S., Hodgson, D., 2010. Boom or bust? a comparative analysis of transient population dynamics in plants. Journal of Ecology 98 (2), 302-311.

Talent, J. A., 2012. Earth and Life. Springer, Dordrecht.

Thiemann, G. W., Iverson, S. J., Stirling, I., 2008. Polar bear diets and arctic marine food webs: insights from fatty acid analysis. Ecological Monographs 78 (4), 591-613.

Turchin, P., 1993. Chaos and stability in rodent population dynamics: evidence from non-linear time-series analysis. Oikos 68 (1), 167-172.

Turchin, P., Hanski, I., 1997. An empirically based model for latitudinal gradient in vole population dynamics. The American Naturalist 149 (5), 842-874.

Turchin, P., Oksanen, L., Ekerholm, P., Oksanen, T., Henttonen, H., 2000. Are lemmings prey or predators? Nature 405 (6786), 562-565.

Vance, R. R., 1978. Predation and resource partitioning in one predator - two prey model communities. The American Naturalist 112 (987), 797-813.

Verhulst, P. F., 1838. Notice sur la loi que la population suite dans son accroissement. correspondence mathematique et physique publiée par A. Quetelet 10, 113-121.

Volterra, V., 1931. Variation and fluctuations of the number of individuals in animal species living together. Animal Ecology, New York, New York, USA, translated from 1928 edition by R. N. Chapman.

Wolf, A., Swift, J. B., Swiney, H. L., Vastano, J. A., 1985. Determining Lyapunov exponents from a time series. Physica D: Nonlinear Phenomena 16 (3), 285-317.
Wrigley, R. E., Hatch, D. R. M., 1976. Arctic fox migrations in Manitoba. Arctic 29 (3), 147-158.

\section{Appendix A Bounded Dynamics of the Primary Model}

Lemma A.1 (Dynamics Bounded Below). Consider the nonautonomous Primary Model given in Equations (2.1)-(2.3) with all parameters strictly positive. Let the initial conditions be $x(0)=x_{0} \geq 0, s(0)=s_{0} \geq 0$, and $y(0)=y_{0} \geq 0$. Then $x(t), s(t)$, and $y(t)$ are bounded below by zero.

Proof. First consider Equation (2.1) to prove that $x(t)$ is bounded below by zero. Note that for $x(t)$ to be negative at some point, there must exist a time, $t^{*}$, such that $x\left(t^{*}\right)=0$ by the Intermediate Value Theorem. However, when $x=0$, we have that $\frac{\mathrm{d} x}{\mathrm{~d} t}=0$ and therefore $x(t)=0 \forall t \geq t^{*}$, contradicting the assumption that $x$ may be negative.

Next we consider Equation (2.3). By the exact same logic, we prove that $y(t)$ is bounded below by zero.

Finally, using Equation (2.2), we get that when $s=0, \frac{\mathrm{d} s}{\mathrm{~d} t}=$ $i(t) \geq 0$ and therefore, $s(t) \geq 0$ for all time.

Lemma A.2 (Dynamics Bounded Above). Consider the nonautonomous Primary Model once again. Let the initial conditions be $x(0)=x_{0} \geq 0, s(0)=s_{0} \geq 0$, and $y(0)=y_{0} \geq 0$. We shall assume that $r(t), k(t)$ and $i(t)$ are bounded non-negative functions with $r(t) \leq r_{\max }, k(t) \leq k_{\max }$, and $i(t) \leq i_{\max }$. Moreover, we assume that $\gamma(t)$ and $\delta(t)$ are positive functions bounded below away from zero, with $\gamma(t) \geq \gamma_{\min }>0$, and $\delta(t) \geq \delta_{\min }>0$. Then there exist positive constants $X, S$, and $Y$ such that $x(t) \leq X, s(t) \leq S$ and $y(t) \leq Y$ for all time.

Proof. The population size of the prey is bounded because for $x>k_{\max }$, we have that $\frac{\mathrm{d} x}{\mathrm{~d} t}<0$. Therefore, $x(t) \leq$ $\max \left\{x_{0}, k_{\max }\right\}=: X$.

Similarly, the population size of the subsidy is bounded because for $s>\frac{i_{\max }}{\gamma_{\min }}$, we have that $\frac{\mathrm{d} s}{\mathrm{~d} t}<0$ and therefore $s(t) \leq$ $\max \left\{s_{0}, \frac{i_{\max }}{\gamma_{\min }}\right\}=: S$.

To examine the dynamics of the predator population, we define

$$
b(t):=\epsilon x(t)+\eta s(t)+y(t) .
$$

Then, from the three differential equations, we see that

$$
\frac{\mathrm{d} b}{\mathrm{~d} t}=\epsilon r x\left(1-\frac{x}{k}\right)+\eta(i-\gamma s)-\delta y .
$$

Now, we note that $\max _{x \geq 0}\left(\epsilon r x\left(1-\frac{x}{k}\right)\right)=\frac{1}{4} \epsilon r_{\max } k_{\max }$ and $\max _{s \geq 0}(\eta(i-\gamma s))=\eta i_{\max }$ to deduce that when

$$
y>\frac{\frac{1}{4} \epsilon r_{\max } k_{\max }+\eta i_{\max }}{\delta_{\min }},
$$

we have that $\frac{\mathrm{d} b}{\mathrm{~d} t}<0$, and so $b(t)$ decreases. Therefore, the maximum value of $b$ is

$$
b_{\max } \leq \epsilon X+\eta S+\max \left\{y_{0}, \frac{\frac{1}{4} \epsilon r_{\max } k_{\max }+\eta i_{\max }}{\delta_{\min }}\right\},
$$


and since $y(t) \leq b(t)$, we get that the predator population size is also bounded with $Y \leq b_{\max }$.

Lemma A.3 (Subsidy Bounded Below Away From Zero). Assume the same conditions as in Lemma A.2. Moreover, assume that $\gamma(t) \leq \gamma_{\max }, i(t) \geq i_{\min }>0$, and $s_{0}>0$. Then $s(t)$ is bounded below away from zero.

Proof. If $i(t)$ is bounded below away from zero, and $\gamma(t)$ is bounded above, then

$$
\frac{\mathrm{d} s}{\mathrm{~d} t} \geq i_{\min }-\gamma_{\max } s-\psi \frac{s}{s+h} y_{\max }
$$

We wish to find conditions under which $\frac{\mathrm{d} s}{\mathrm{~d} t} \geq 0$, and therefore we solve

$$
\begin{aligned}
& 0 \leq i_{\min }-\gamma_{\max } s-\psi \frac{s}{s+h} y_{\max } \\
& 0 \geq \varsigma(s) .
\end{aligned}
$$

Noting that $\varsigma(s)=\gamma_{\max } s^{2}+\left(\psi y_{\max }+\gamma_{\max } h-i_{\min }\right) s-i_{\min } h$ is a positive parabola and that $s(0)<0$, we deduce that there exists $s^{*}>0$ such that $\varsigma\left(s^{*}\right)=0$ and therefore $s(s) \leq 0$ whenever $s \in\left[0, s^{*}\right]$. Consequently, $\frac{\mathrm{d} s}{\mathrm{~d} t} \geq 0$ for $s$ in this range. Hence, $s(t) \geq \min \left\{s_{0}, s^{*}\right\}$ and thus bounded below away from zero.

Note that if $\left.\frac{\mathrm{d} s}{\mathrm{~d} t}\right|_{s=0} \geq i_{\min }>0$ and if $s_{0}=0$ then after an arbitrarily small amount of time, $s(t)$ is strictly positive and so Lemma A.3 can be applied.

\section{Appendix B Bounded Dynamics of the $n$-Patch Model}

Lemma B.1 (Dynamics Bounded Below). Consider the nPatch Model given by Equations (2.4)-(2.6), with positive parameters given in Section 2 and non-negative initial conditions $x^{(j)}(0)=x_{0}^{(j)}, s^{(j)}(0)=s_{0}^{(j)}$, and $y^{(j)}(0)=y_{0}^{(j)}$. Then $x^{(j)}(t), s^{(j)}(t), y^{(j)}(t) \geq 0$ for all time.

Proof. The proof that $x^{(j)}(t) \geq 0$ and $s^{(j)}(t) \geq 0$ follows in a similar way to Lemma A.1.

Let $T$ denote the set of times for which any of $y^{(j)}(t)$ 's is negative. That is

$$
T=\left\{t \geq 0 \mid \exists j \text { with } y^{(j)}(t)<0\right\} .
$$

If $T \neq \emptyset$, then by the Fundamental Axiom of the real numbers, $T$ must have an infimum, which we denote by $t^{*}=\inf T$. At $t=$ $t^{*}$, we have the onset of negativity, and therefore $y^{(j)}\left(t^{*}\right) \geq 0$ for all $j$, and there exists some $\ell$ with $y^{(\ell)}\left(t^{*}\right)=0$ and $\left.\frac{\mathrm{d} y^{(\ell)}}{\mathrm{d} t}\right|_{t=t^{*}}<0$.

Now,

$$
\left.\frac{\mathrm{d} y^{(\ell)}}{\mathrm{d} t}\right|_{t=t^{*}}=\sum_{m \neq \ell} \lambda^{(m, \ell)} \alpha^{(m, \ell)}\left(t^{*}\right) y^{(m)}\left(t^{*}\right) \geq 0,
$$

giving a contradiction.

Thus, $x^{(j)}(t), s^{(j)}(t)$, and $y^{(j)}(t)$ all remain non-negative.

Lemma B.2 (Dynamics Bounded Above). Consider the nPatch Model again. Suppose that the prey growth rates, $r^{(j)}(t)$, the prey carrying capacities, $k^{(j)}(t)$, and the subsidy input rates, $i^{(j)}(t)$, are all bounded above. Suppose further that the predator mortality rates, $\delta^{(j)}(t)$, and the predator migration rates, $\alpha^{(j, \ell)}(t)$, are bounded below away from zero. Then the prey populations, $x^{(j)}(t)$, the subsidy populations, $s^{(j)}(t)$, and the predator populations, $y^{(j)}(t)$, are all bounded above.

Proof. In a similar manner to Lemma A.2, the prey population sizes, $x^{(j)}(t)$ 's, are bounded above by $\max \left\{x_{0}^{(j)}, k_{\max }^{(j)}\right\}$, and the subsidy population sizes, $s^{(j)}(t)$ 's, are bounded above by $\max \left\{s_{0}^{(j)}, i_{\max }^{(j)} / \gamma_{\min }^{(j)}\right\}$.

Let

$$
B=\sum_{j=1}^{n} \epsilon x^{(j)}(t)+\eta s^{(j)}(t)+y^{(j)}(t),
$$

so that

$$
\begin{aligned}
\frac{\mathrm{d} B}{\mathrm{~d} t}= & \sum_{j=1}^{n} \epsilon r^{(j)} x^{(j)}\left(1-\frac{x^{(j)}}{k^{(j)}}\right)+\eta\left(i^{(j)}-\gamma^{(j)} s^{(j)}\right) \\
& -\left(\delta^{(j)}+\sum_{\ell \neq j} \alpha^{(j, \ell)}\right) y^{(j)}+\sum_{\ell \neq j} \lambda^{(\ell, j)} \alpha^{(\ell, j)} y^{(\ell)} \\
= & \sum_{j=1}^{n} \epsilon r^{(j)} x^{(j)}\left(1-\frac{x^{(j)}}{k^{(j)}}\right)+\eta\left(i^{(j)}-\gamma^{(j)} s^{(j)}\right) \\
& -\left(\delta^{(j)}+\sum_{\ell \neq j}\left(1-\lambda^{(j, \ell)}\right) \alpha^{(j, \ell)}\right) y^{(j)} \\
\leq & \sum_{j=1}^{n} \frac{1}{4} \epsilon r_{\max }^{(j)} k_{\max }^{(j)}+\eta i_{\max }^{(j)}-\left(\delta_{\min }^{(j)}+\sum_{\ell \neq j}\left(1-\lambda^{(j, \ell)}\right) \alpha_{\min }^{(j, \ell)}\right) y^{(j)} .
\end{aligned}
$$

Suppose that $B$ is unbounded above. Since $x^{(j)}(t)$ and $s^{(j)}(t)$ are bounded above, it follows that $\sum_{j} y^{(j)}(t)$ must be unbounded above. Consequently, at some time, $t$, it must be true that

$$
\sum_{j=1}^{n} y^{(j)}(t)>\frac{\sum_{j=1}^{n} \frac{1}{4} \epsilon r_{\max }^{(j)} k_{\max }^{(j)}+\eta i_{\max }^{(j)}}{\min _{j}\left(\delta_{\min }^{(j)}+\sum_{\ell \neq j}\left(1-\lambda^{(j, \ell)}\right) \alpha_{\min }^{(j, \ell)}\right)} .
$$

Note that the right hand side of this inequality is a constant. When Inequality (B.4) holds, we have

$$
\sum_{j=1}^{n}\left(\delta_{\min }^{(j)}+\sum_{\ell \neq j}\left(1-\lambda^{(j, \ell)}\right) \alpha_{\min }^{(j, \ell)}\right) y^{(j)}>\sum_{j=1}^{n} \frac{1}{4} \epsilon r_{\max }^{(j)} k_{\max }^{(j)}+\eta i_{\max }^{(j)},
$$

which when combined with Inequality (B.4), shows that $\frac{\mathrm{d} B}{\mathrm{~d} t}<0$ and so $B$ must be decreasing. Therefore, $B$ must be bounded above. Consequently, since $\sum_{j} y^{(j)}(t)<B(t)$, we deduce that $\sum_{j} y^{(j)}(t)$ is bounded above. Therefore, each of the $y^{(j)}(t)$ 's must be bounded above as well.

Hence, we have proven that the $x^{(j)}(t)$ 's, $s^{(j)}(t)^{\prime}$ 's, and the $y^{(j)}(t)$ 's are all bounded above. 